\title{
Stability Analysis of a Single-Degree-of-Freedom Mechanical Model with Distinct Critical Points: I. Bifurcation Theory Approach
}

\author{
Dimitrios S. Sophianopoulos \\ Department of Civil Engineering, University of Thessaly, Volos, Greece \\ Email: dimsof@civ.uth.gr
}

Received November 7, 2012; revised December 10, 2012; accepted December 24, 2012

\begin{abstract}
The buckling and post-buckling response of a single-degree-of-freedom mechanical model is re-examined in this work, within the context of nonlinear stability and bifurcation theory. This system has been reported in pioneer as well as in more recent literature to exhibit all kinds of distinct critical points. Its response is thoroughly discussed, the effect of all parameters involved is extensively examined, including imperfection sensitivity, and the results obtained lead to the important conclusion that the model is possibly associated with the butterfly singularity, a fact which will be validated by the contents of a companion paper, based on catastrophe theory.
\end{abstract}

Keywords: Mechanical Models; Nonlinear Stability; Distinct Critical Points; Bifurcation Theory; Singularities

\section{Introduction}

The role of distinct critical points, namely symmetric (stable or unstable) branching points, asymmetric branching points and limit points, has been recognized to be of paramount importance in the General Theory of Elastic Stability [1-3]. Moreover, the distinct branching points, arising in perfect conservative discrete systems, have been shown to be essentially one degree of freedom phenomena [4]. Their study, as well as the effect of initial imperfections on their evolution and on the overall system's response can be performed by applying either the Nonlinear Stability and Bifurcation Theory [5] or the Catastrophe Theory [6].

Both theories start from the formulation of the total potential energy function of the system, but proceed afterwards to different directions. The former uses the exact system potential and its derivatives to evaluate equilibrium paths, critical points and their stability and produces results concerning the particular system dealt with. On the other hand, the latter theory utilizes the structure of universal unfoldings of the potential and classifies them to the so-called elementary and higher order Catastrophes, which have a priori known features and properties. Hence, qualitative general (universal) solutions are established, producing a better insight into nonlinear phenomena, such as discontinuities, singularities and instabilities.

The above two theories have their advantages and dis- advantages, which - among other causes - are originated from the number of degrees of freedom (generalized active coordinates) and the number of control parameters and thus on the resulting complexity of the total potential energy function. For systems with one or two degrees of freedom in particular, there can be cases where both Theories are challenged by sometimes immense mathematical difficulties; then, only combining the application of both theories may lead to a true understanding of the stability response of such a system [7].

In the context of the above remark, the present work deals with the nonlinear stability analysis of a mechanical model with a single active variable (degree of freedom) and with four control parameters, which has been reported in the literature to exhibit all kinds of distinct critical points $[8,9]$. It is found that the gradual introduction of control parameters (including initial imperfection) leads to all kinds of elementary singularities for a single variable, and that the fully imperfect system is possibly associated with the butterfly catastrophe. Analysis and proof of these findings via Catastrophe Theory will be given in the 2nd part of this work in a companion paper.

\section{Single Degree of Freedom Mechanical Model-Geometry, Description and General Buckling Equations}

We consider the single-degree-of-freedom (D.O.F.) mechanical model depicted in Figure 1, which, as men- 
tioned in the Introduction, may exhibit all kinds of distinct critical points [9]. The system comprises of a weightless rigid bar of length $\ell$, partially pinned at its base via a linear rotational spring of stiffness $c$; its tip is connected to an inclined linear extensional spring of stiffness $k$, with the angle of inclination kept always constant and equal to $\alpha$, since the other end of the spring may freely slide along equally inclined supports. The tip of the bar is acted upon by a gravitational force $P$. The single degree of freedom characterizing the deformation of the system is hence the rotation $\theta$ of the bar, which in general is considered imperfect, by introducing an initial rotation $\varepsilon$. At this stage all springs are considered unstressed. For the model to be realistic, the values of angles $\alpha, \varepsilon$ and $\theta$ are limited within the following intervals:

$$
(0 \leq \alpha \leq \pi),(-\pi / 2 \leq \varepsilon \leq \pi / 2),(-\pi<\theta<\pi) .
$$

If under the action of $\mathrm{P}$ the system deforms as shown in Figure 1, its total potential energy $V_{T}$ will be equal to the sum of the strain energy $U$ and the work of the external force $\Omega$, given in the most general case by:

$$
U=\frac{1}{2} k \delta_{A}^{2}+\frac{1}{2} M(\theta-\varepsilon) \text {. }
$$

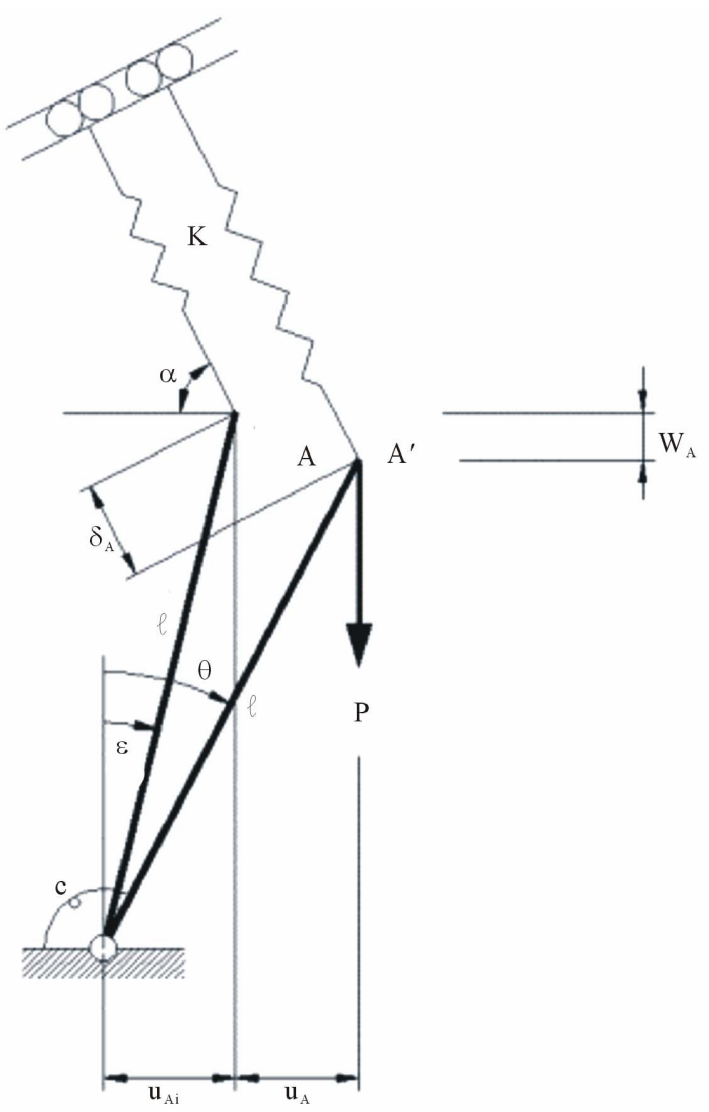

Figure 1. Geometry and sign convention of the single D.O.F. model considered.

$$
\Omega=-P w_{A} .
$$

where $M$ is the moment developed by the rotational spring at the base, $\delta_{A}$ the change of length of the extensional spring and $w_{A}$ the vertical displacement of the tip, which are equal to

$$
\begin{aligned}
& M=c(\theta-\varepsilon) . \\
& w_{A}=\ell(\cos \varepsilon-\cos \theta) . \\
& \delta_{A}=\ell\{\sin (a-\varepsilon)-\sin (a-\theta)\} .
\end{aligned}
$$

Hence, the total potential energy function of the system yields

$$
\begin{aligned}
V_{T}= & U+\Omega=\frac{1}{2} k \ell^{2}[\sin (a-\varepsilon)-\sin (a-\theta)]^{2} \\
& +\frac{1}{2} c(\theta-\varepsilon)^{2}-P \ell(\cos \varepsilon-\cos \theta) .
\end{aligned}
$$

Introducing the following dimensionless parameters

$$
\beta=\frac{c}{k \ell^{2}}, \lambda=\frac{P}{k \ell} .
$$

the nondimesionalized potential takes the form

$$
\begin{aligned}
\bar{V}_{T}= & \frac{V_{T}}{k \ell^{2}}=\frac{1}{2}[\sin (a-\varepsilon)-\sin (a-\theta)]^{2} \\
& +\frac{1}{2} \beta(\theta-\varepsilon)^{2}-\lambda(\cos \varepsilon-\cos \theta) .
\end{aligned}
$$

Within the context of nonlinear elastic stability and bifurcation theory, equilibria, critical points and their corresponding stability will be sought thereafter, by studying the nature of the derivatives of the energy function given above. In doing this, the equilibrium equation is acquired thorough the principle of the stationary value of the potential, i.e.

$$
\lambda_{E}=\frac{[\sin (a-\varepsilon)-\sin (a-\theta)] \cos (a-\theta)+\beta(\theta-\varepsilon)}{\sin \theta} .
$$

The stability of non-critical equilibrium points depend on the sign of the second variation of the total potential at these points, which is computed from expression (9) and is equal to

$$
\begin{aligned}
& V_{\vartheta \vartheta}=\left[\frac{\mathrm{d}^{2} \bar{V}_{T}}{\mathrm{~d} \vartheta^{2}}\right]_{\lambda=\lambda_{E}} \\
& =\cos 2(a-\theta)+\sin (a-\varepsilon) \sin (a-\theta) \\
& +\beta-\frac{[\sin (a-\varepsilon)-\sin (a-\theta)] \cos (a-\theta)+\beta(\theta-\varepsilon)}{\tan \theta}
\end{aligned}
$$

Finally, the stability of critical points will be established by the sign of higher order derivatives of the potential energy function.

It should be noted that the values of $\beta=0$ imply the 
absence of the rotational base spring, and equivalently the presence of a pure hinge. On the other hand, very large values of $\beta$ can be associated by either a very rigid base or by a very "light" spring at the tip.

Evidently, the response of the single D.O.F. model dealt with depends on the variation of four control parameters, namely of $\lambda, a, \beta$ and $\varepsilon$. The last three may be considered as perturbations of the fully perfect system $(\alpha$ $=\varepsilon=\beta=0$ ), the study of which will be essential for the detailed model stability analysis. The individual and combined variation of parameters $\alpha, \beta$ and $\varepsilon$ lead to the need of studying the response of the following system cases:

Case 1: $\alpha=\varepsilon=0, \beta \neq 0$

Case 2: $\beta=\varepsilon=0, \alpha \neq 0$

Case 3: $\alpha=\beta=0, \varepsilon \neq 0$

Case 4: $\alpha=0, \beta$ and $\varepsilon \neq 0$

Case 5: $\beta=0, \alpha$ and $\varepsilon \neq 0$

Case 6: $\varepsilon=0, \alpha$ and $\beta \neq 0$.

Case 7: The general imperfect system $(\alpha, \beta, \varepsilon \neq 0)$

In these Cases, the inclusion of imperfection $\varepsilon$ is a "severe" perturbation, while of $\alpha$ and/or $\beta$ "mild" ones [2,5]. The fully perfect system (being in fact a mechanism) as well as all the aforementioned system Cases will be studied in detail in what follows.

It should be noted that Cases $1-3$ involve two control parameters, Cases 4 - 6 three and Case 7 four control parameters, while for all Cases there exists only a single active variable, i.e. rotation $\theta$.

\section{Bifurcational Analysis, Numerical Results and Discussion}

\subsection{The "Fully" Perfect System $(\alpha=\beta=\varepsilon=0)$}

Denoting for simplicity

$$
V=\bar{V}_{T} \text { and } V_{1}=\frac{\mathrm{d} \bar{V}_{T}}{\mathrm{~d} \theta},
$$

from expressions (8)-(10) it is deduced that

$$
\begin{aligned}
& V=-\lambda(1-\cos \theta)+\frac{\sin \theta^{2}}{2}, \\
& V_{1}=-\lambda \sin \theta+\cos \theta \sin \theta . \\
& \lambda_{E}=\cos \theta . \\
& {\left[\frac{\mathrm{d}^{2} V}{\mathrm{~d} \theta^{2}}\right]_{\lambda=\lambda_{E}}=-\frac{1}{4} \csc \theta(3 \sin \theta-\sin 3 \theta) .}
\end{aligned}
$$

The equilibrium path of the model, from Equation (11b) (in solid line), as well as the second variation of the potential, from Equation (11c) (in dashed line), are illustrated in Figure 2.

As expected, the path possesses a profound symmetry, is unstable within the interval $(-\pi, \pi)$ and furthermore is

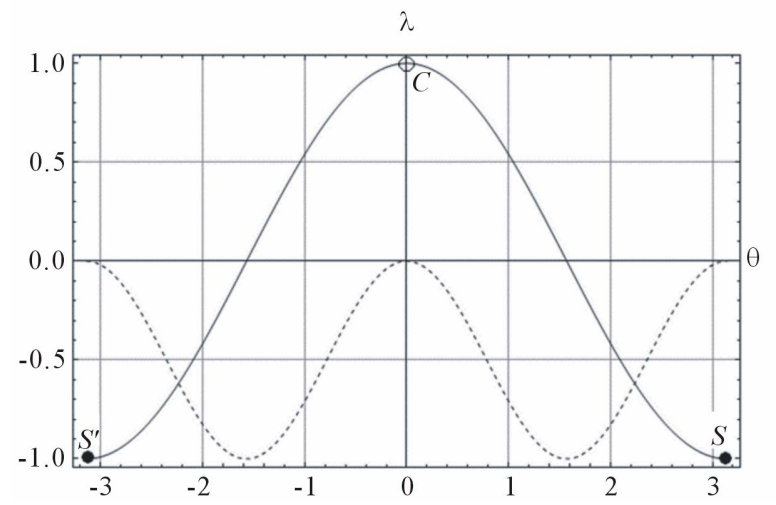

Figure 2. Equilibrium path and 2nd variation of the potential for the "fully" perfect system.

associated with an unstable symmetric branching point $\mathrm{C}$ (for $\lambda=1$ ) as well as with two limit points $S$ and $S^{\prime}$ (for $\lambda$ $=-1$ and $\theta=\pi, \theta=-\pi$ respectively). This model configuration will be perturbed thereafter, according to the definition of the preceding Cases.

\subsection{Case 1: $\alpha=\varepsilon=0, \beta \neq 0$}

Mildly perturbing the "fully perfect" system, by introducing variations of parameter $\beta$, i.e. by accounting for some semi-rigidity of the base support, while the spring at the top is horizontal at the left of the origin, the corresponding expressions for the potential, the equilibrium equation and the 2 nd variation of the potential at the equilibria are given by:

$$
\begin{aligned}
& V=\frac{\beta \theta^{2}}{2}-\lambda(1-\cos \theta)+\frac{(\sin \theta)^{2}}{2} \\
& V_{1}=\beta \theta-\lambda \sin \theta+\cos \theta \sin \theta \\
& \lambda_{E}=\cos \theta+\beta \theta \csc \theta \\
& {\left[\frac{\mathrm{d}^{2} V}{\mathrm{~d} \theta^{2}}\right]_{\lambda=\lambda_{E}}=\frac{1}{2}(-1+2 \beta+\cos 2 \theta-2 \beta \theta \cot \theta) .}
\end{aligned}
$$

Since no initial imperfection or any rotational disturbance is introduced, it is anticipated that the symmetry of the equilibrium paths will be preserved.

The possible existence of bifurcation points is sought by evaluating the intersection of the paths given in Equation (12b) with the $\lambda$-axis. This is achieved by calculating the limit of $\lambda_{E}$ as $\theta$ tends to zero, which gives

$$
\lim _{\vartheta \rightarrow 0} \lambda_{E}=\lambda_{c}=1+\beta .
$$

This implies that a symmetric branching point (of yet unknown stability) always exists, while additional critical points (namely limit point ones) of the equilibrium path can be found by seeking additional roots (with respect to $\beta$ ) of the 2nd variation given in expression (12c) for $\theta \neq 0$. In doing this, it is found that 


$$
\beta=\beta_{s}(\theta)=\frac{\sin ^{2} \theta}{1-\theta \cot \theta} .
$$

Thus, the locus of the limit points is given by the following function (a product of substituting relation (14) in Equation (12b)):

$$
\lambda_{S, S^{\prime}}=\cos \theta+\frac{\theta \sin \theta}{1-\theta \cot \theta} .
$$

Exploring the nature of the analytical function given in Expression (15) in conjunction with the limiting value of $\beta_{s}(\theta)$ from Equation (14), for $\theta \rightarrow 0$, being equal to

$$
\beta_{c r}=\lim _{\theta \rightarrow 0} \beta_{s}=3
$$

it is presumed that the two additional (symmetric) limit points and the bifurcation point are degenerated to a single branching point at $\beta=3, \lambda=4$. At this critical situation there exists a change of stability of the earlier defined bifurcation point $C$ from unstable symmetric to stable symmetric. All the above findings are schematically shown in Figure 3.

As far as the stability of equilibria is concerned, the paths are unstable between $S$ and $S^{\prime}$, and stable beyond. This is valid for $\beta<3(\lambda<4)$. Above this value the paths are stable throughout. From a bifurcational point of view, this specific "mild" perturbation of the "fully" perfect system preserves symmetry and up to a specific value of $\beta$ also the nature and stability of paths and critical points.

Beyond this value, the system becomes totally stable. This observation may be perceived from the corresponding bifurcation diagram of Figure 4.

\subsection{Case 2: $\beta=\varepsilon=0, \alpha \neq 0$}

For this particular Case, being also a "mild" perturbation of the "fully" perfect system described in Section 3.1, the introduction of the variation of the angular spring-inclination parameter $\alpha$ is expected to alter the nature of the branching point (which will be always present since the system has no initial imperfection). In the same manner as in Case 1, the expressions for $V, V_{1}, \lambda_{E}$ and

$$
\left[\frac{\mathrm{d}^{2} V}{\mathrm{~d} \theta^{2}}\right]_{\lambda=\lambda_{E}}
$$

are found equal to:

$$
\begin{aligned}
& V=\sin ^{2} \frac{\theta}{2}[1-2 \lambda \cos (2 \alpha-\theta)], \\
& V_{1}=\cos [a-\theta](\sin a-\sin [a-\theta])-\lambda \sin \theta . \\
& \lambda_{E}=\cos [a-\theta] \cos \left[a-\frac{\theta}{2}\right] \sec \frac{\theta}{2} . \\
& {\left[\frac{\mathrm{d}^{2} V}{\mathrm{~d} \theta^{2}}\right]_{\lambda=\lambda_{E}}=} \\
& -\frac{1}{4} \csc \theta(2 \sin 2 a+\sin [2 a-3 \theta]-3 \sin [2 a-\theta]) .
\end{aligned}
$$

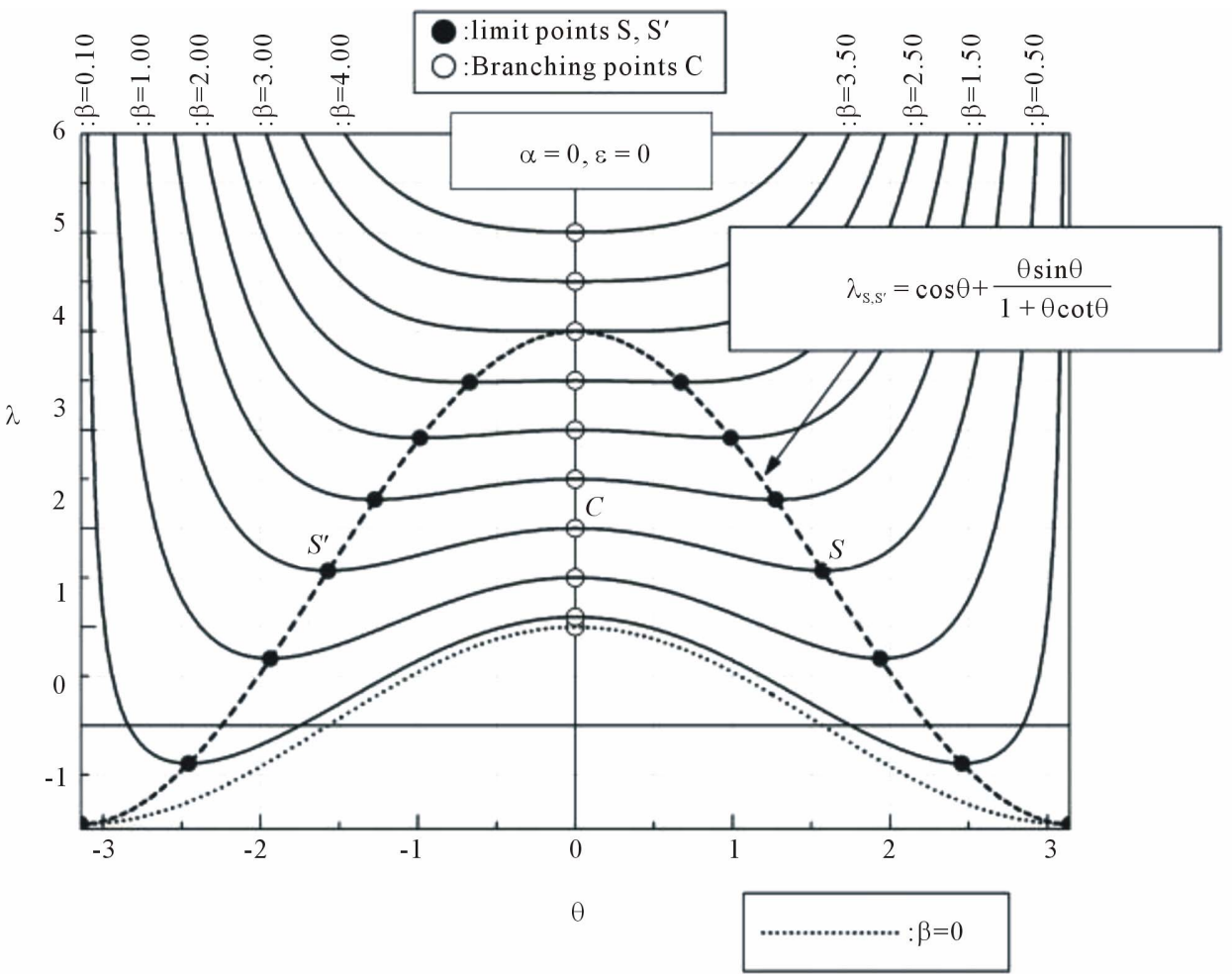

Figure 3. Equilibrium paths and locus of critical points for system Case 1 (the dotted line refers to the "fully" perfect system). 


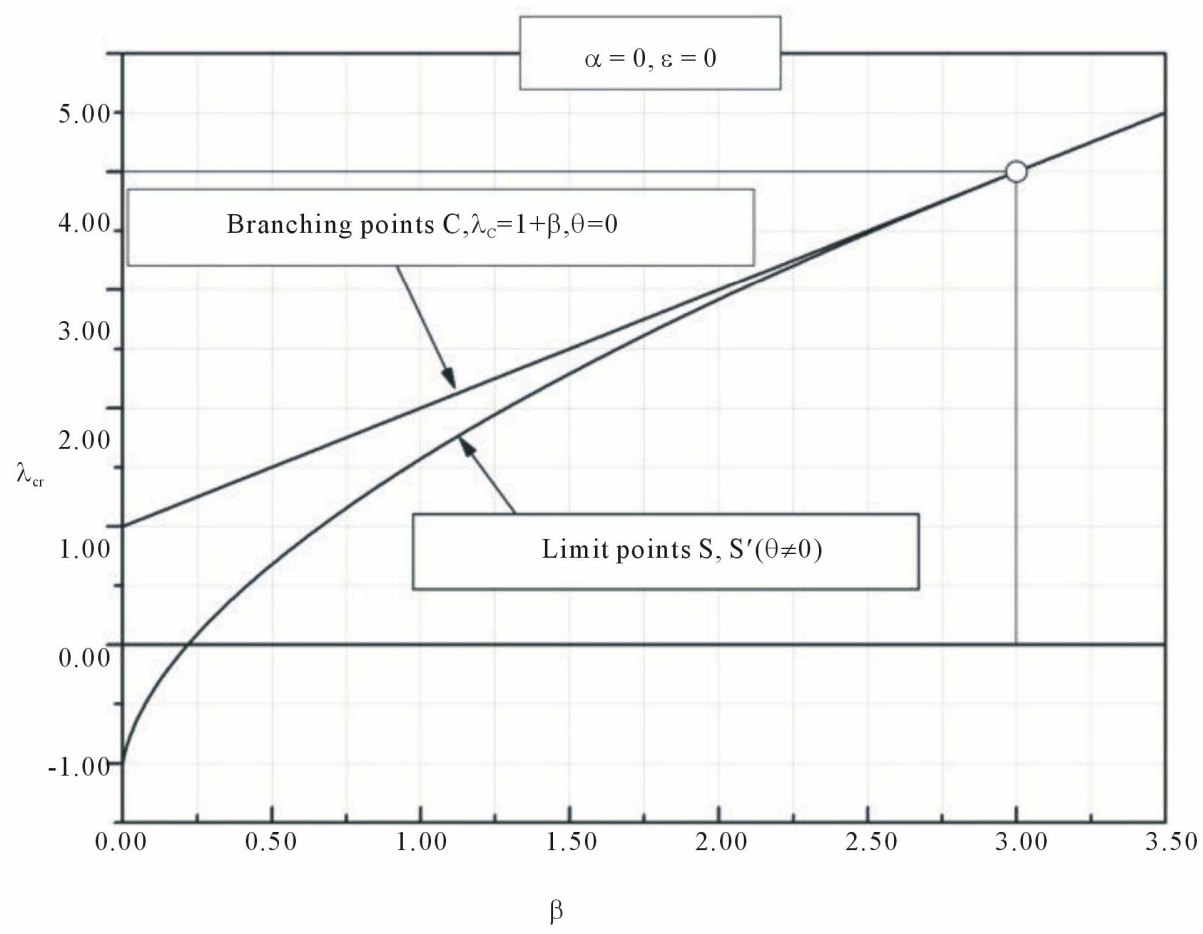

Figure 4. Bifurcation diagram of the system of Case 1.

Contrary to the previous Case, where parameter $\beta$ acts linearly, the spring-inclination parameter $\alpha$ of the foregoing Case is always included in trigonometric expressions. This may act as an unsurpassed obstacle in obtaining analytical expression for the loci of critical points, especially limit-point ones.

The branching point, being the intersection of the equilibrium paths given is Expression (17b) with the $\lambda$-axis, is computed via Equation (17b), producing the following result:

$$
\lim _{\theta \rightarrow 0} \lambda_{E}=\lambda_{C}=\cos ^{2} \alpha .
$$

Aiming to quantitatively explore the nature of the equilibrium paths and their critical points, the paths for two characteristic values of $\alpha(0.3<\pi / 2$ and $2.4>\pi / 2)$ are computed and presented graphically in Figure 5.

Both paths exhibit an asymmetric branching point $\left(C_{1}\right.$ and $C_{2}$ respectively) as well as two limit points $\left(S_{1}, S_{1}^{\prime}\right.$ and $S_{2}, S_{2}^{\prime}$ respectively), the latter computed numerically. The paths are unstable between the limit points and stable beyond those, while-as expected from expression (17b) - they have asymptotes at $\theta= \pm \pi$. After these initial estimates, one may gain a more comprehensive picture of the nature of the system's response for the foregoing Case, from the contents of Figure 6, where equilibrium paths for various values of $\alpha$ are presented.

It is readily perceived that for every value of parameter $\alpha$ two limit points and an asymmetric branching point appear. Starting from small values of $\alpha$ and increasing it, the branching point is moved from its original place (for the fully perfect system) downwards, until it reaches the origin for $a=\pi / 2$. Similarly, starting from large values of $\alpha$ (near $\pi$ ) and decreasing them, identical results are obtained. The transition of the corresponding limit points (as well as of the branching points) is shown in the bifurcation diagram of Figure 7 that follows.

The load corresponding to the 1 st limit point $S$, for values of $\alpha \leq \pi / 2$ shows an increasing pattern, becoming maximum at $\alpha=\pi / 2$; same response is observed for $S^{\prime}$ but for $\pi / 2 \leq \alpha<\pi$, while for $\alpha=\pi / 2$ it coincides with the corresponding branching point (this being also true for $S$ for $0 \leq \alpha \leq \pi / 2)$. The shape of the diagrams in Figure 7 clearly reveals one individual and two dependent cusps at $\pi / 2$, with some indication of existence of three symmetric folds $[6,10]$.

\subsection{Case 3: $\beta=\alpha=0, \varepsilon \neq 0$ (Simplest Imperfect System)}

Since initial angular imperfection is introduced, this Case will be surely associated with symmetry-breaking bifurcations. It is the simplest imperfect system, it constitutes a severe perturbation of the "fully" perfect system and for this Case it is valid that:

$$
\lambda_{E}=\cos \theta-\cot \theta \sin \varepsilon .
$$

Typical equilibrium paths for various values of the initial imperfection $\varepsilon$ (positive or negative) and for $\theta$ ranging from $-\pi$ to $\pi$ are shown in Figures 8-11. 


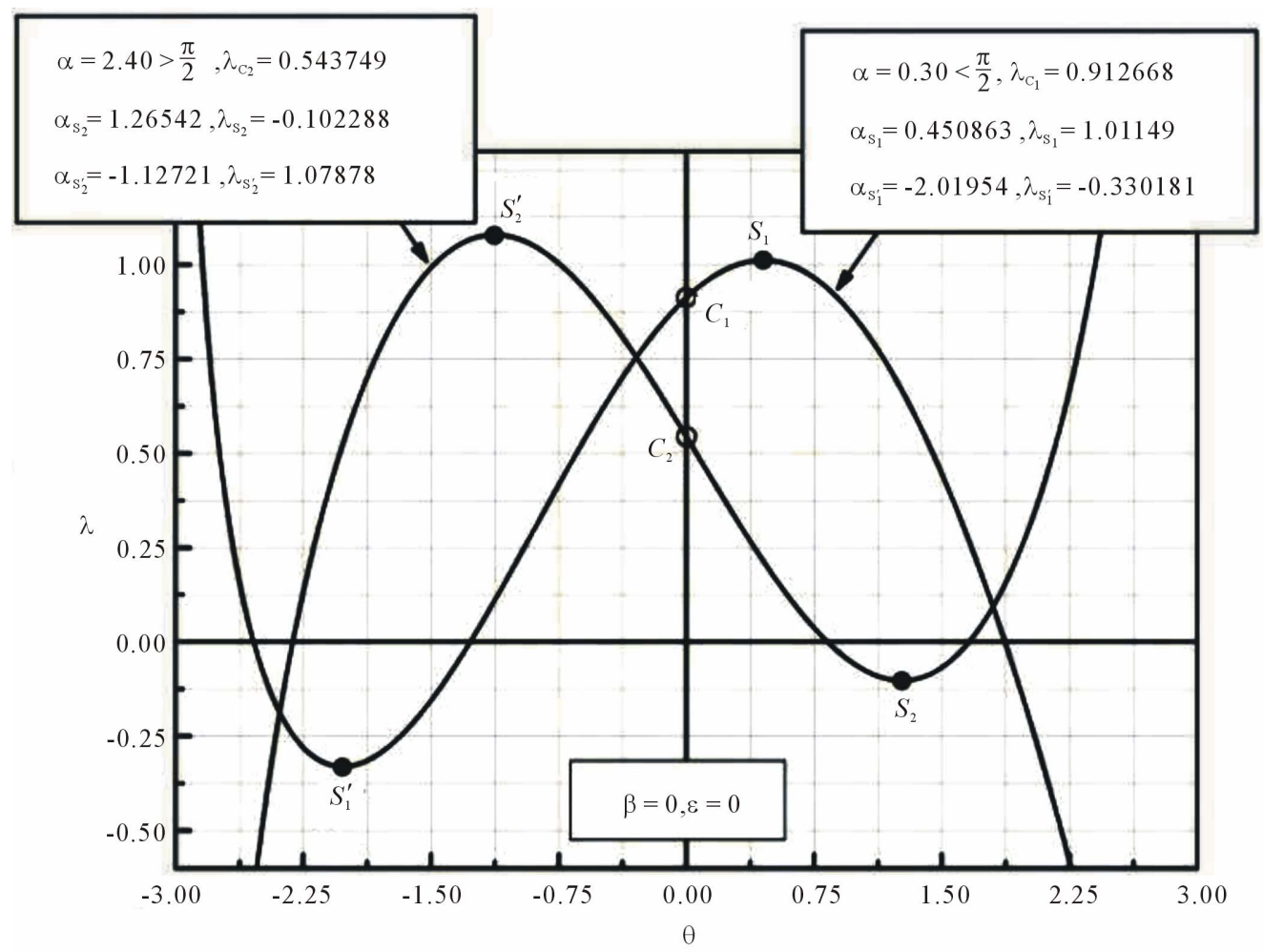

Figure 5. Equilibrium paths with their critical points for Case 2 and two characteristic values of parameter $\alpha$.

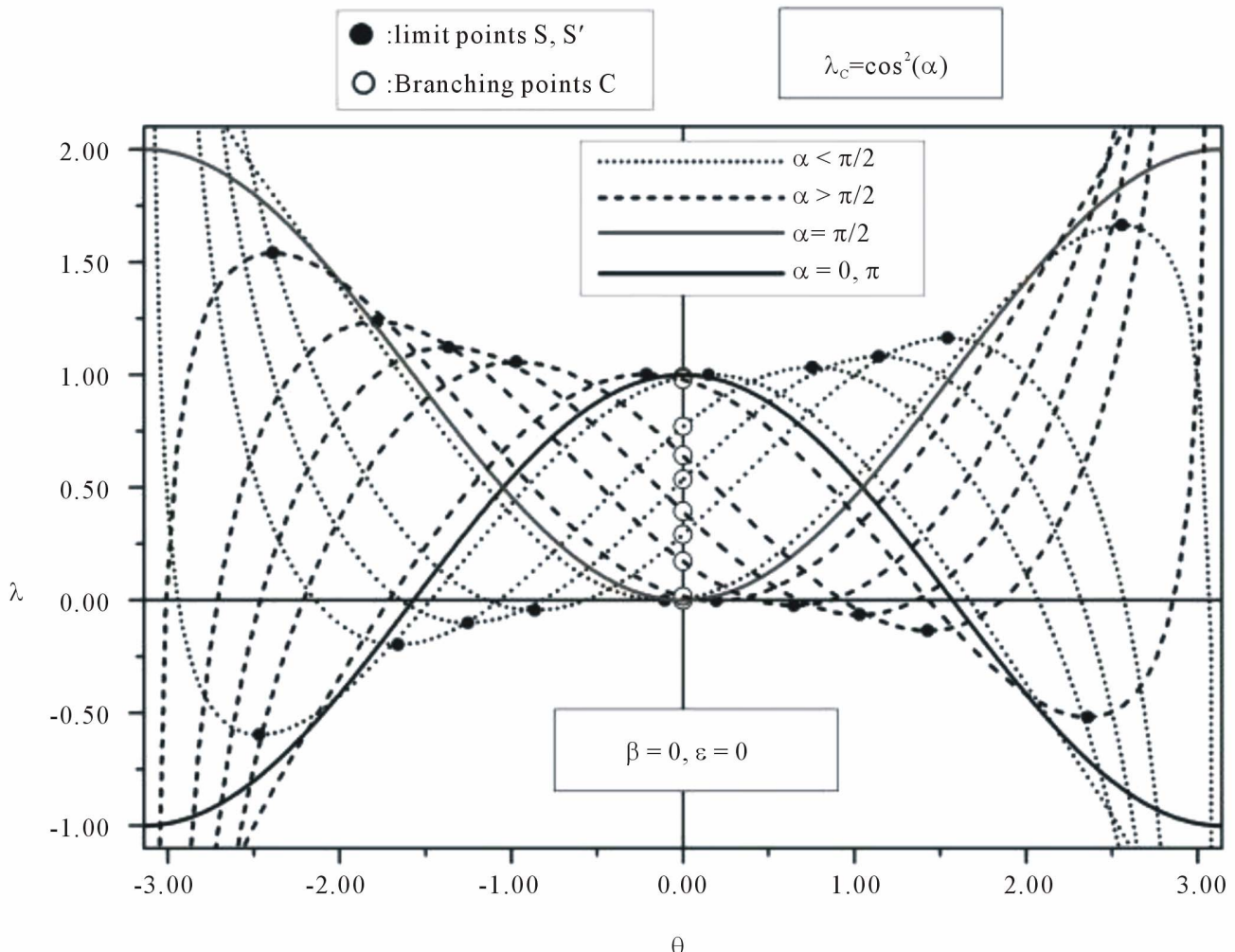

Figure 6. Equilibrium paths for Case 2 and various values of $\alpha$. 


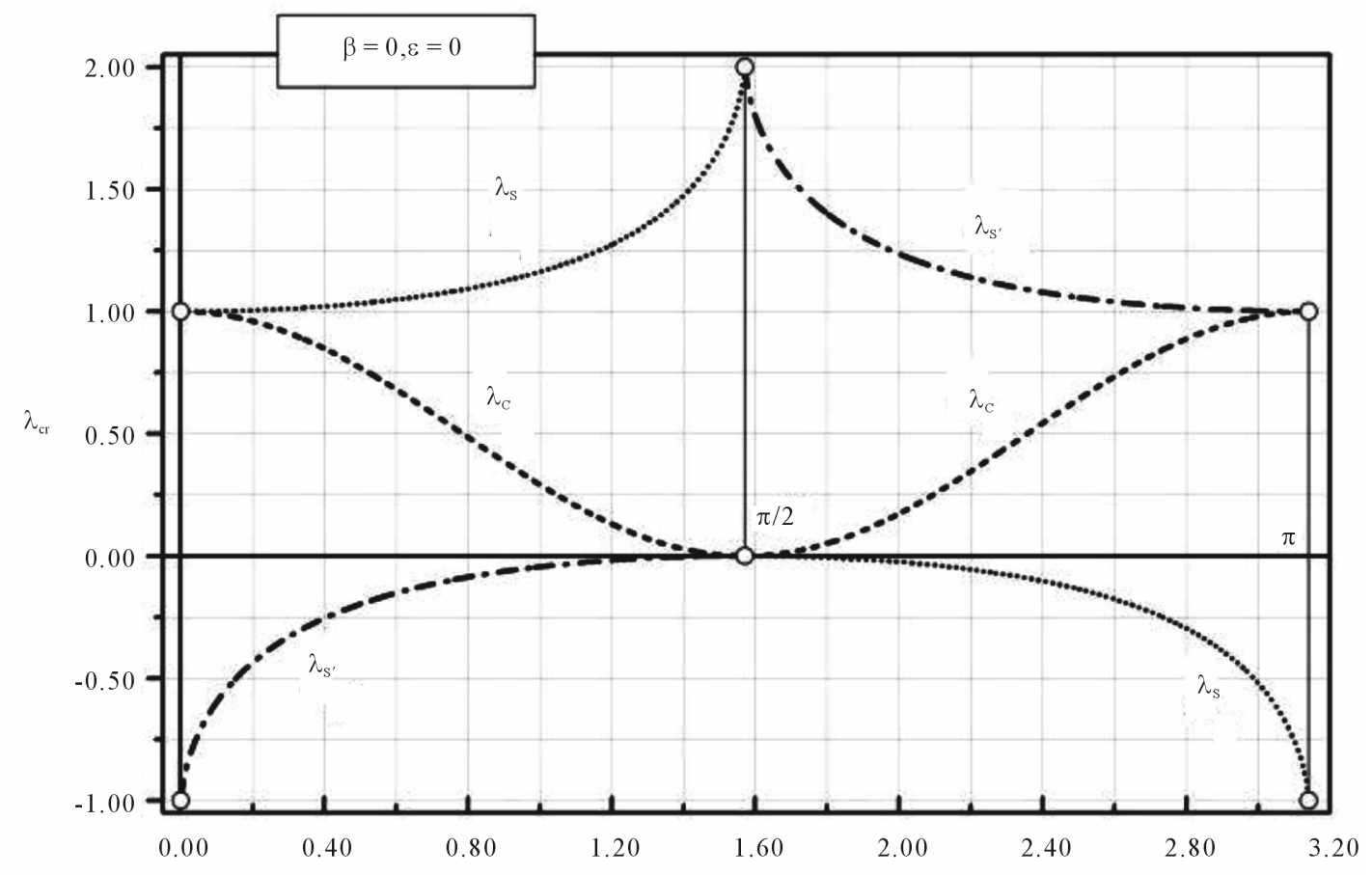

Figure 7. Bifurcation diagram of the system's Case 2.

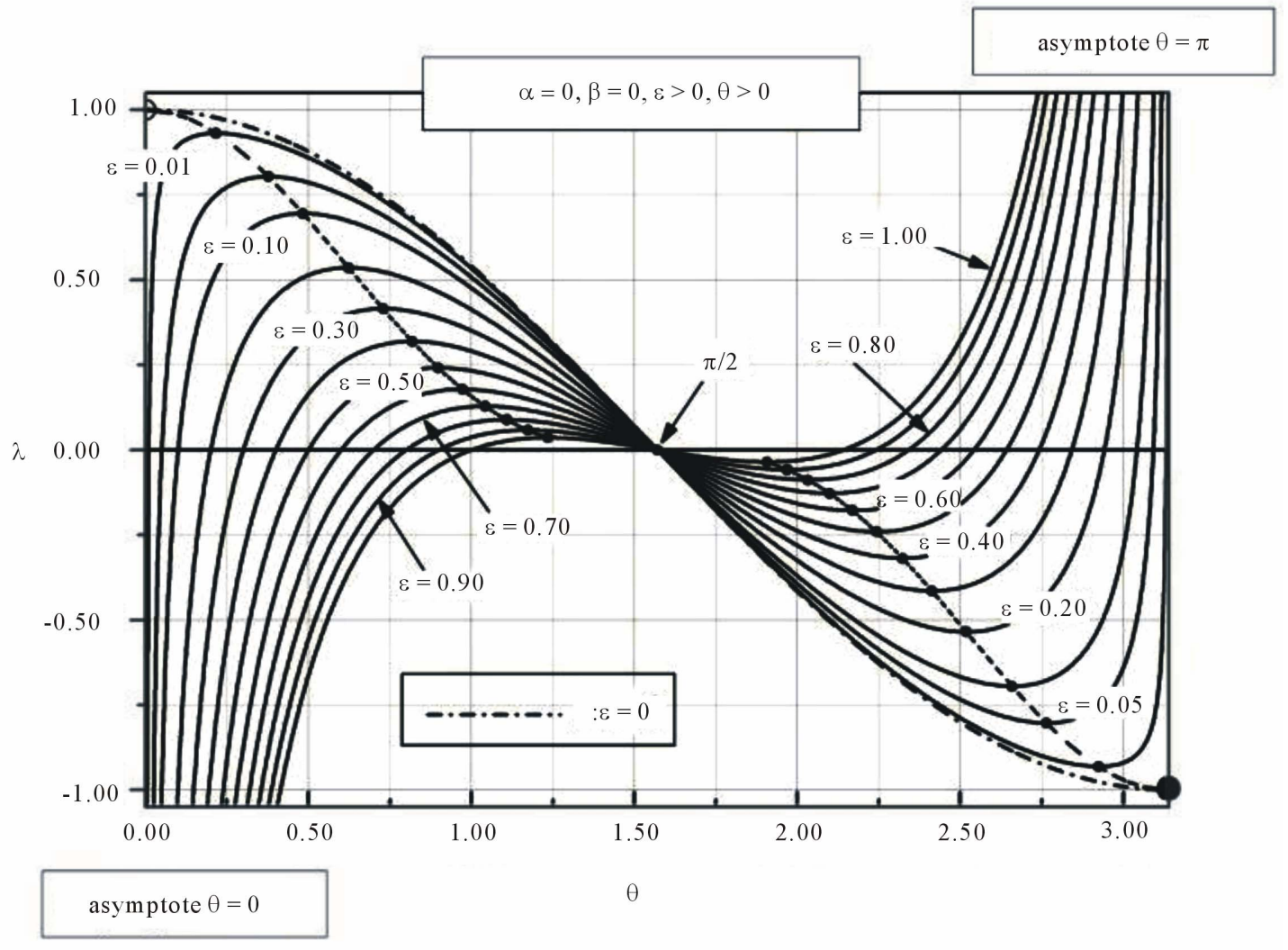

Figure 8. Equilibrium paths and critical point of Case 3 for $\varepsilon>0$ and $0<\theta<\pi$. 


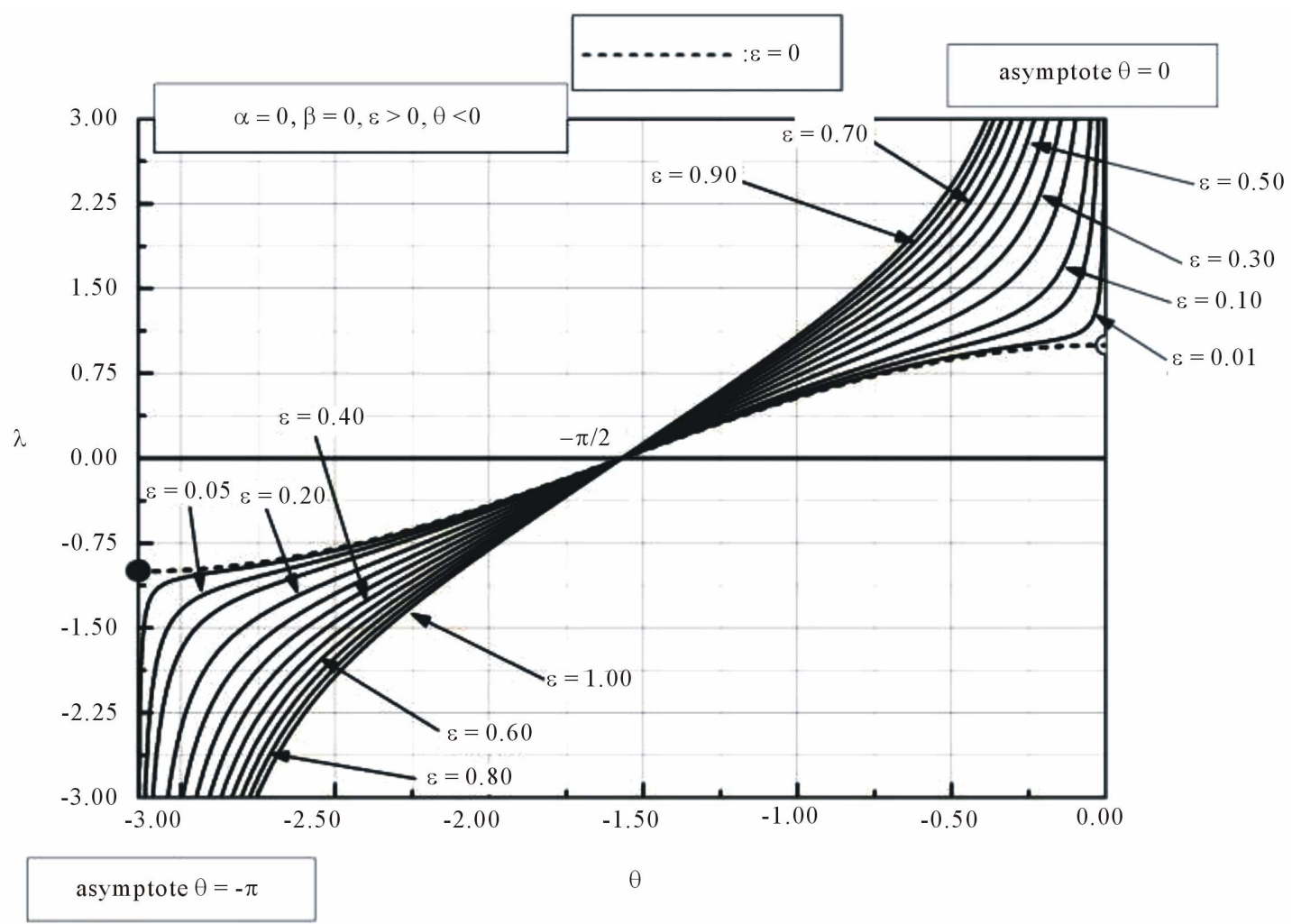

Figure 9. Equilibrium paths and critical point of Case 3 for $\varepsilon>0$ and $-\pi<\theta<0$.

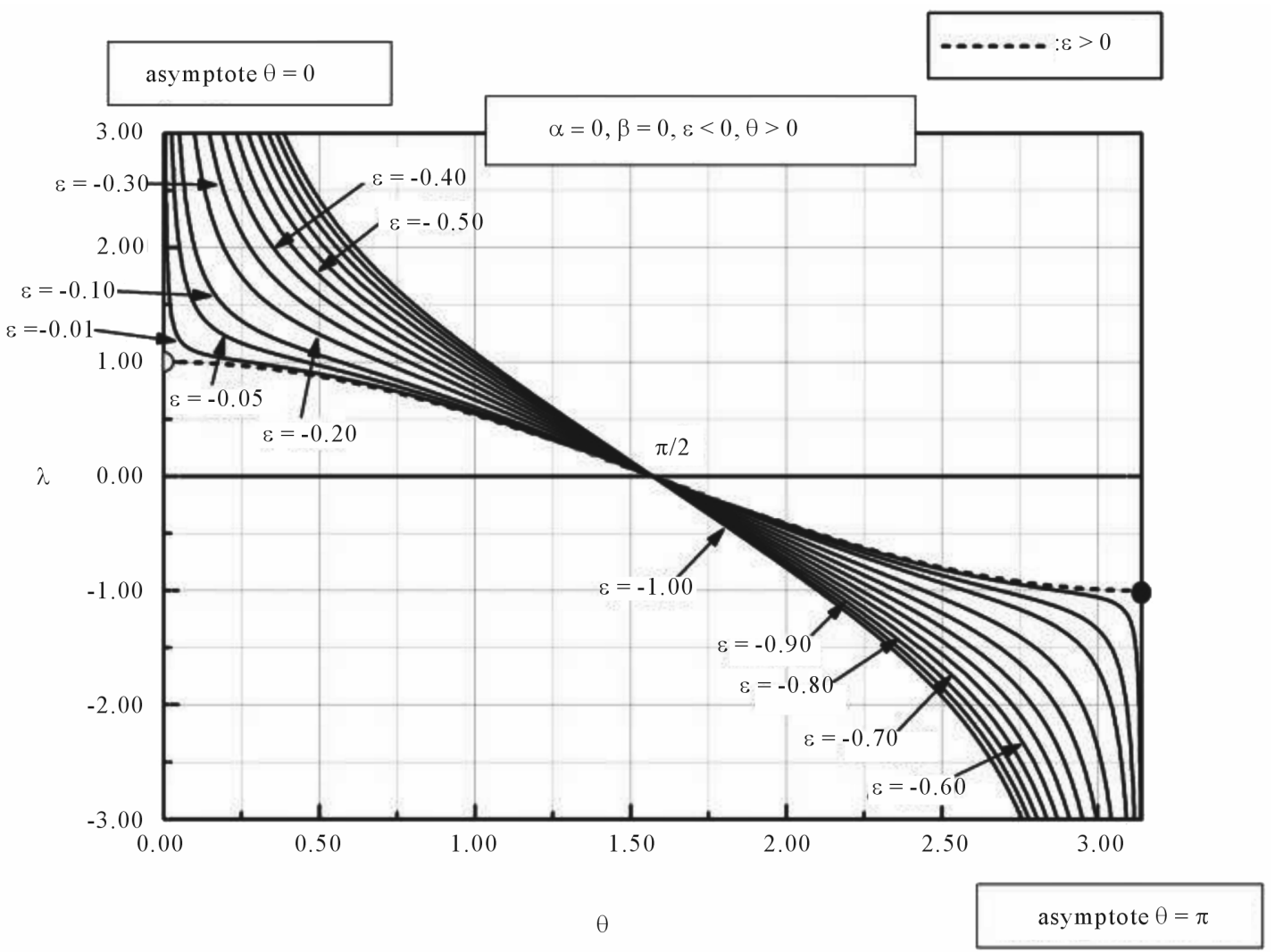

Figure 10. Equilibrium paths and critical point of Case 3 for $\varepsilon<0$ and $0<\theta<\pi$. 


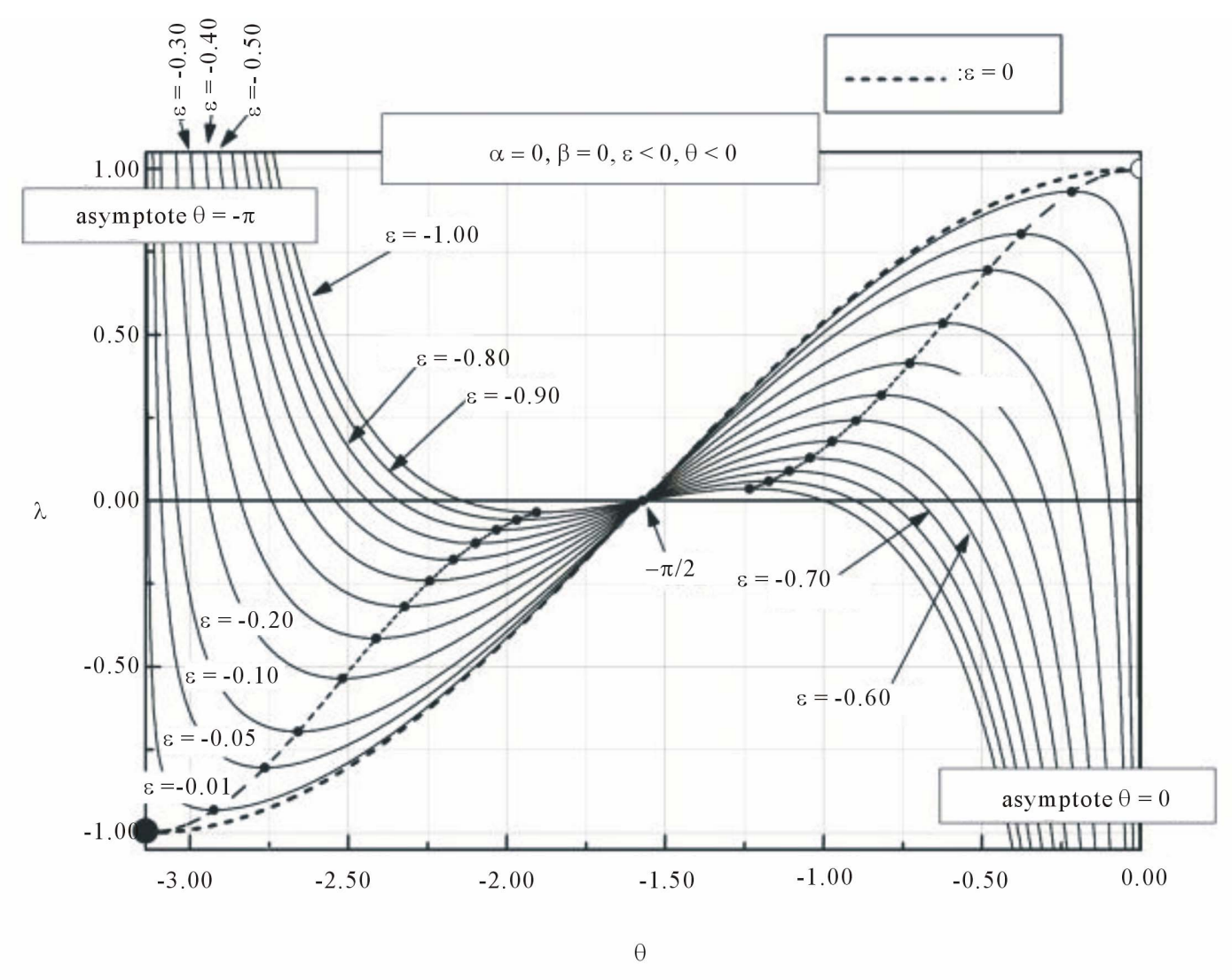

Figure 11. Equilibrium paths and critical point of Case 3 for $\varepsilon<0$ and $-\pi<\theta<0$.

Due to symmetry breaking, primary and complementary equilibrium paths are produced, leading to the birth of one or two limit points. Setting the 2 nd variation of the total potential energy equal to zero, these limit points appear for

$$
\lambda=\lambda_{S}=\cos ^{3} \theta,
$$

regardless of the value of $\varepsilon$. Moreover, the diagram of perturbed bifurcations for this Case is drawn and presented in Figure 12. From this Figure one may perceive the evolution of the critical points of the "fully" perfect system $(C, S$ and $S$ '- see Figure 2) as $\varepsilon$ varies.

More specifically, branching point $C$ (unstable symmetric) transitions into a limit point of ascending equilibrium path, while one of the two limit points is preserved and the other disappears; depending on the sign of the initial imperfection the limit point that is preserved is the one arising for $\theta$ having the same sign as $\varepsilon$. Additionally, the cusp singularity for $C$ is clearly present along with its symmetrical one for $S$ and $S$.

\subsection{Case 4: $\alpha=0, \beta, \varepsilon \neq 0$ (Severe Perturbation of the System of Case 1)}

Introducing initial angular imperfection $\varepsilon$ for the system Case 1 (which was found symmetric_-see Figure 3), once again symmetry breaking bifurcations are expected. For the foregoing Case one may write that:

$$
\begin{aligned}
& V=\frac{\beta(\theta-\varepsilon)^{2}}{2}+\frac{(\sin \theta-\sin \varepsilon)^{2}}{2}-\lambda(\cos \varepsilon-\cos \theta) . \\
& \lambda_{E}=\frac{\beta(\theta-\varepsilon)+\cos \theta(\sin \theta-\sin \varepsilon)}{\sin \theta} .
\end{aligned}
$$

Typical equilibrium paths for three characteristic values of $\beta$ and positive $\varepsilon$ are shown in Figures 13-15. For negative $\varepsilon$ the paths are symmetric with respect to the vertical axis. In these Figures all critical points are depicted (for both the perturbed and unperturbed system).

For values of $\beta$, for which the corresponding perfect system exhibits - beyond the unstable symmetric branching point - two limit points, the complementary paths of the imperfect system exhibit a limit point, originated from the perturbation of one of the limit points of the corresponding perfect system; the related primary paths exhibit two limit points, one being the perturbation of the branching point of the perfect system and the other being the perturbation of the remaining limit point of the perfect system.

Contrary, for $\beta \geq 3$ (existence of only one critical point which is a stable symmetric branching one) the one and 


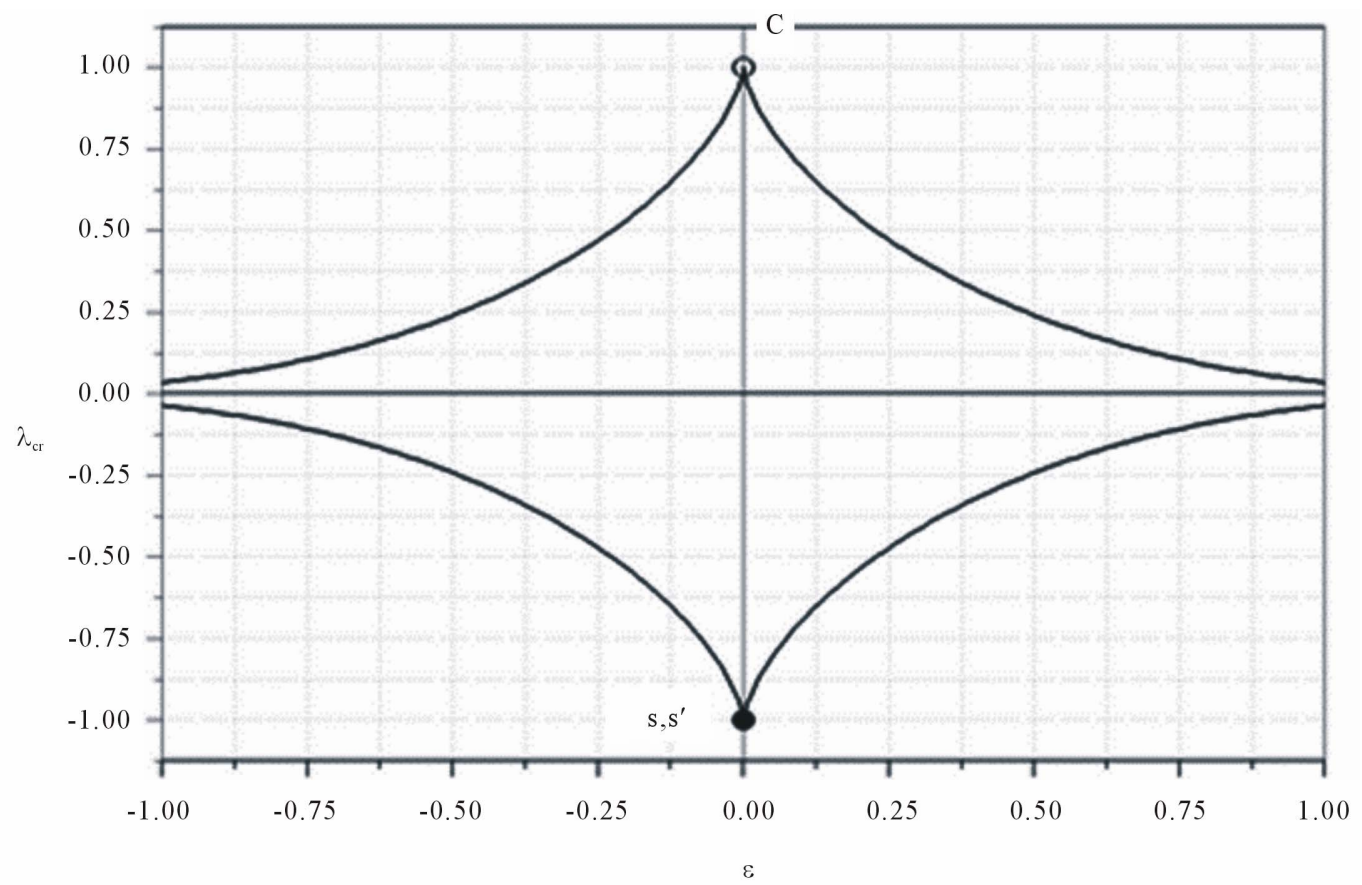

Figure 12. Diagram of perturbed bifurcations for Case 3.

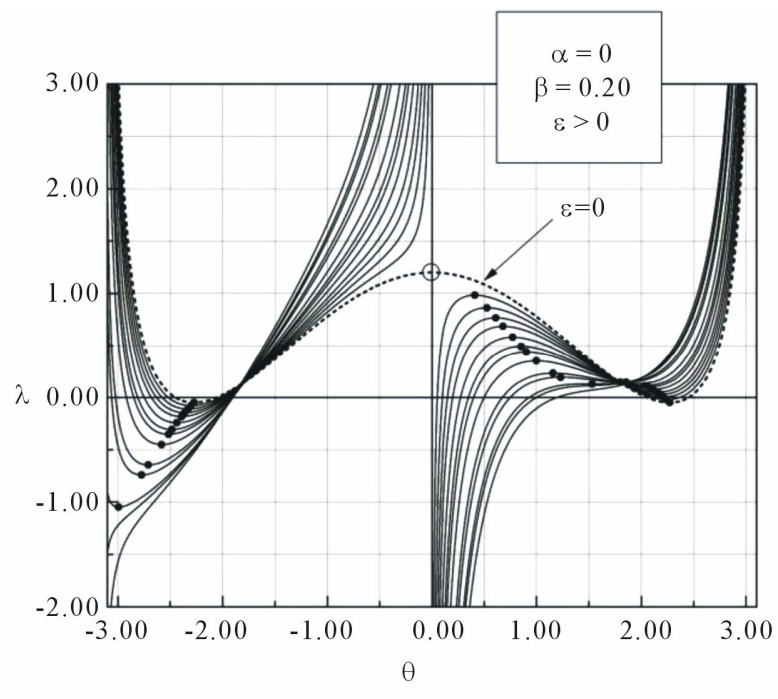

Figure 13. Equilibrium paths for Case $3, \beta=0.20$ and $\varepsilon>0$.

only limit point of the perturbed system arises on the complementary paths and is in fact a perturbation of the branching point, while all primary paths are monotonically rising.

Moreover, where primary paths process two limit points, there always exists a critical value of $\varepsilon$ where a cutoff point (coincidence of these limit points-horizontal tangent) is revealed. For larger $\varepsilon$, these paths become monotonically rising. The above findings are graphically shown in the representative diagrams of perturbed bifurcations of Figure 16.

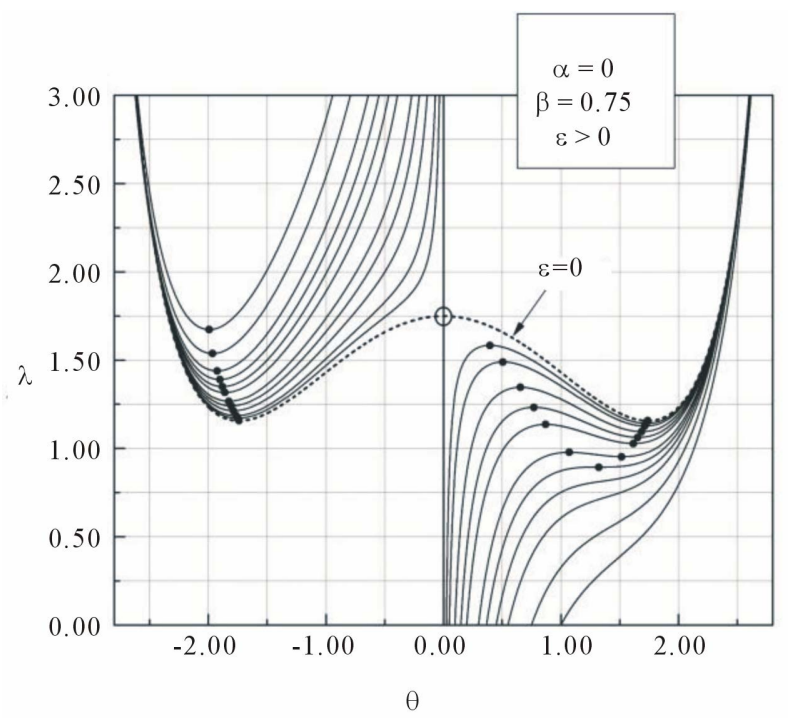

Figure 14. Equilibrium paths for Case $3, \beta=0.75$ and $\varepsilon>0$.

From these graphs it is evident that the system Case dealt with in this section, possessing a three dimensional control space $(\lambda, \beta, \varepsilon)$ and one state variable $(\theta)$ is associated with the swallowtail singularity $[2,6]$.

\subsection{Case 5: $\beta=0, \alpha, \varepsilon \neq 0$ (Severe Perturbation of the System of Case 2)}

Due to absence of symmetry in the corresponding perfect system (of Case 2), the effect of the introduction of the initial imperfection $\varepsilon$ will depend mainly on the salient 


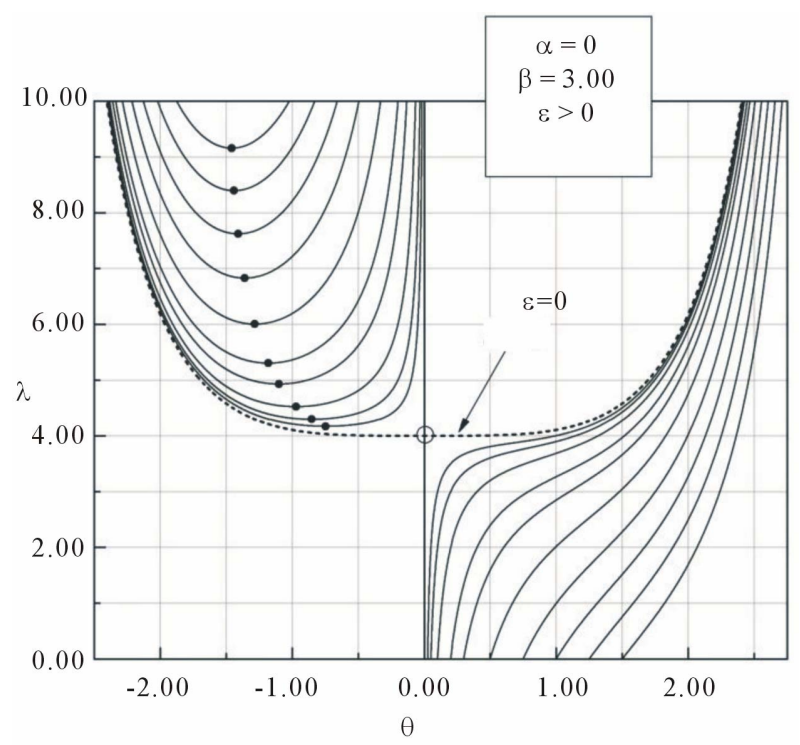

Figure 15. Equilibrium paths for Case 3, $\beta=3.00$ and $\varepsilon>0$.
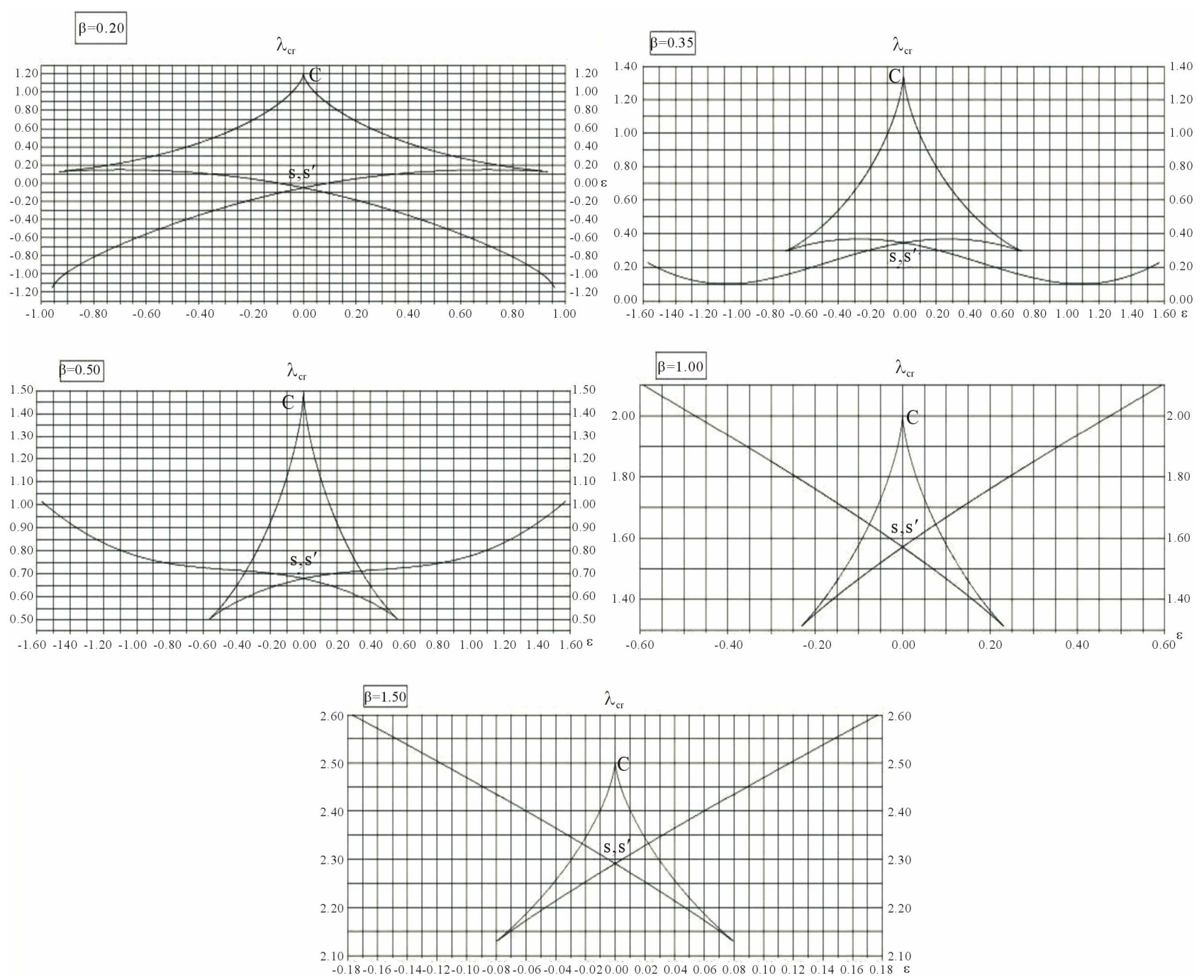

Figure 16. Diagrams of perturbed bifurcations of Case 4 and various values of $\beta$. 
comes a continuous function of $\theta$ (within the interval $-\pi$, $\pi$ ) and coincides with the related path of the unperturbed system, as shown in Figure 17, for $\alpha=1.25$.

Additionally, in the graphs presented in Figures 18 and 19, equilibrium paths and critical points are depicted, for a constant small value of $\varepsilon$ (either positive or negative) and various values of parameter $\alpha$.

Interestingly enough, when $\varepsilon=2 \alpha$ the system for $\theta=$ $-\pi$ exhibits a critical point for $\lambda=-\cos ^{2} \alpha$, i.e. for $\lambda=-\lambda_{c}$, where $\lambda_{c}$ is the branching load of the corresponding perfect system. For a better insight on the evolution of critical points, diagrams of perturbed bifurcations and corresponding details are presented in Figure 20 (for $\alpha<\pi / 2$ ) and Figure 21 (for $\alpha>\pi / 2$ ).

The critical points shown in these Figures are denoted as follows:
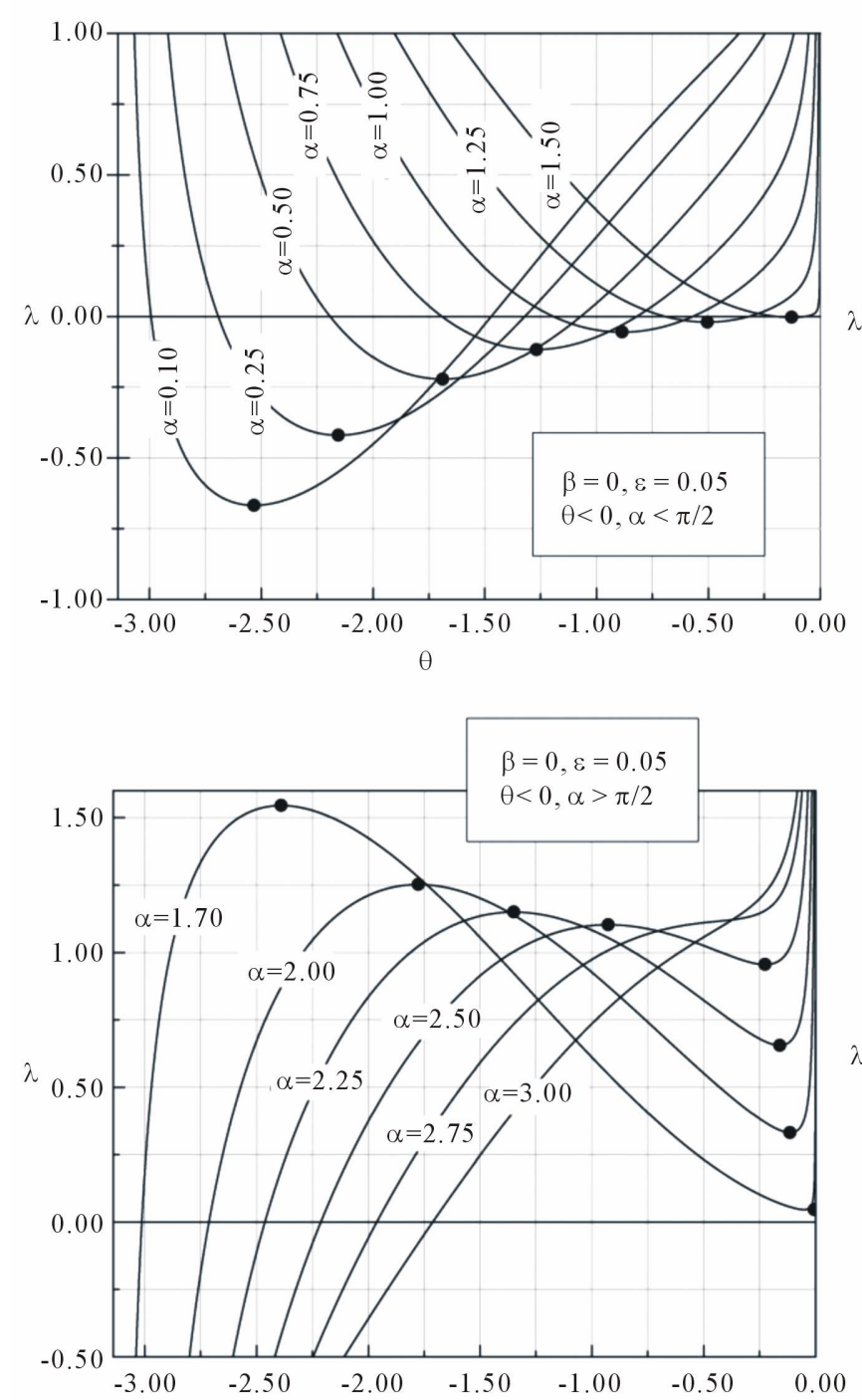

$\theta$

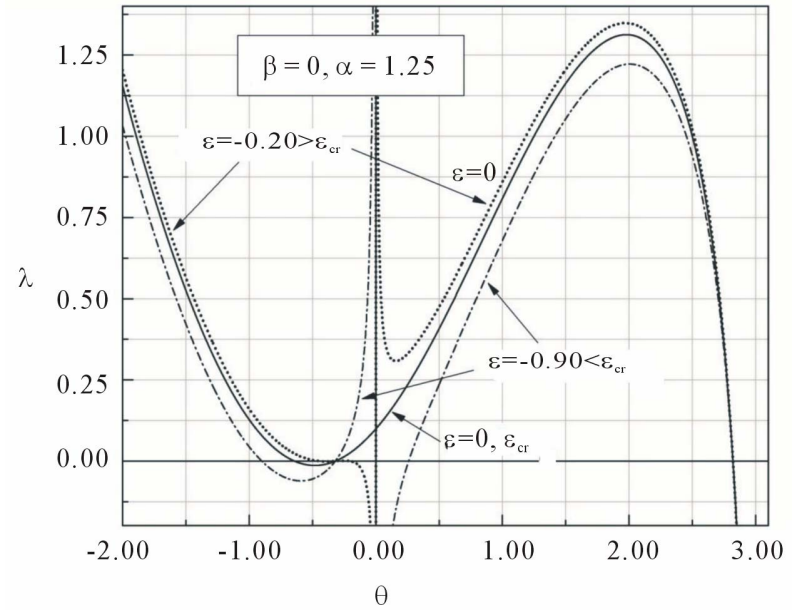

Figure 17. Equilibria for the special case $\varepsilon=\varepsilon c r(\alpha=1.25)$.
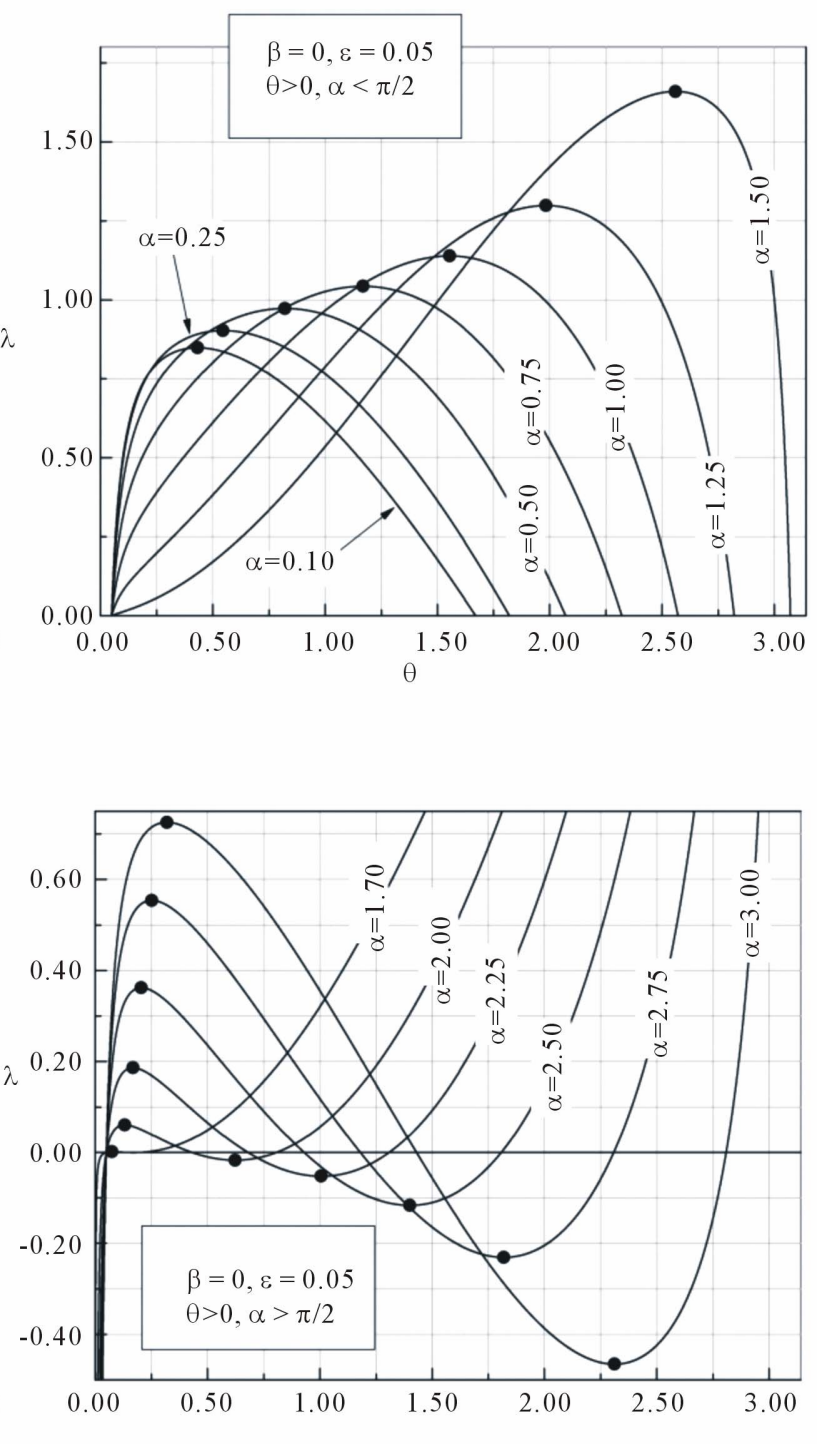

$\theta$

Figure 18. Equilibrium paths and critical points for Case 5, with $\varepsilon=0.05$ and various values of $\alpha$. 

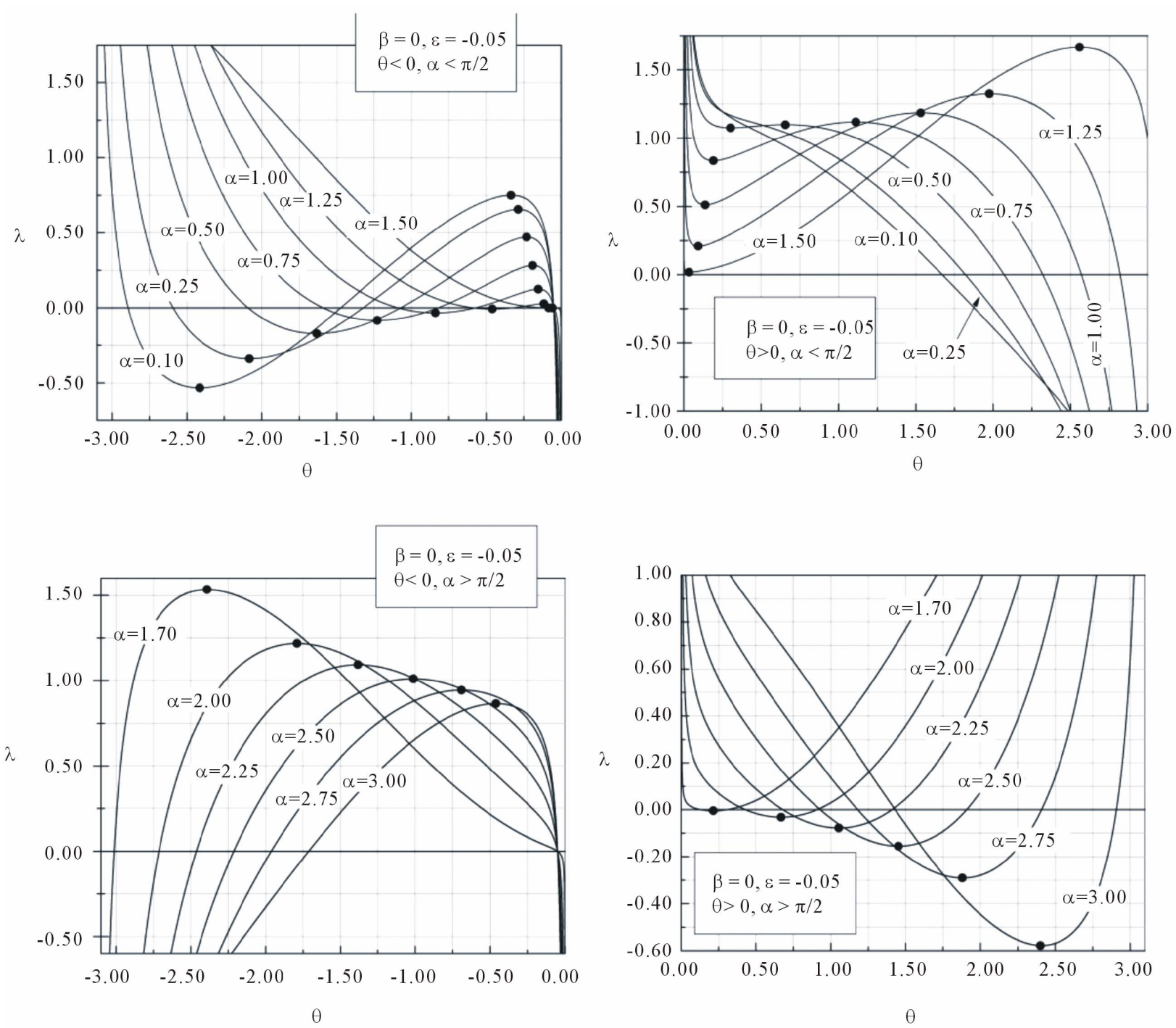

Figure 19. Equilibrium paths and critical points for Case 5, with $\varepsilon=-0.05$ and various values of $\alpha$.

$C, S, S^{\prime}$ : Branching point and limit points of the corresponding unperturbed (perfect) system.

$C^{\prime}$ : Critical (limit) point for $\varepsilon=2 \alpha$ and $\lambda_{c r}=-\lambda_{c}$.

$I_{1}, I_{2}$ : Cut-off (hysteresis) points with equal and opposite critical loads (tilts).

$I_{3}$ : Cut-off point for $\lambda=0$.

$C^{\prime \prime}$ : Branching point for $\varepsilon=2 \alpha-\pi$ and $\lambda=\lambda_{c}$.

It can be seen that for certain values of $\alpha$ (but not close to $\pi / 2$ ) the system exhibits tilted cusps, while for other values of $a$ these cusps are degenerated, with the progressive appearance of multiple folds of axial symmetry. Such phenomena have been reported in the literature [6] to be closely related to the swallowtail as well as to the butterfly singularity.

\subsection{Case 6: $\varepsilon=0, \alpha, \beta \neq 0$}

The system in this Case evolves from either Case 1 or
Case 2 by introducing a "mild" perturbation, namely $\alpha$ or $\beta$ respectively. In doing this, we get

$$
\begin{aligned}
& V=\frac{\beta \theta^{2}}{2}-\lambda(1-\cos \theta)+\frac{1}{2}[\sin \alpha-\sin (\alpha-\theta)]^{2} . \\
& \lambda_{E}=\csc \theta(\beta \theta+\cos [a-\theta](\sin a-\sin [a-\theta])) .
\end{aligned}
$$

Two sets of equilibrium paths are given below, by varying $a$ for some specific values of $\beta$ in Figure 22 and for specific values of $\alpha$ and varying $\beta$ in Figure 23 .

From these plots it is deduced that as $\beta$ increases, the equilibrium paths are shifted upwards, while for increasing $\alpha$ the plots are rotated anticlockwise (for $\alpha<\pi / 2$ ) or clockwise (for $\alpha>\pi / 2$ ). Moreover, and for every combination of parameters $a$ and $\beta$, the system exhibits an asymmetric branching point, corresponding to the following value of loading: 

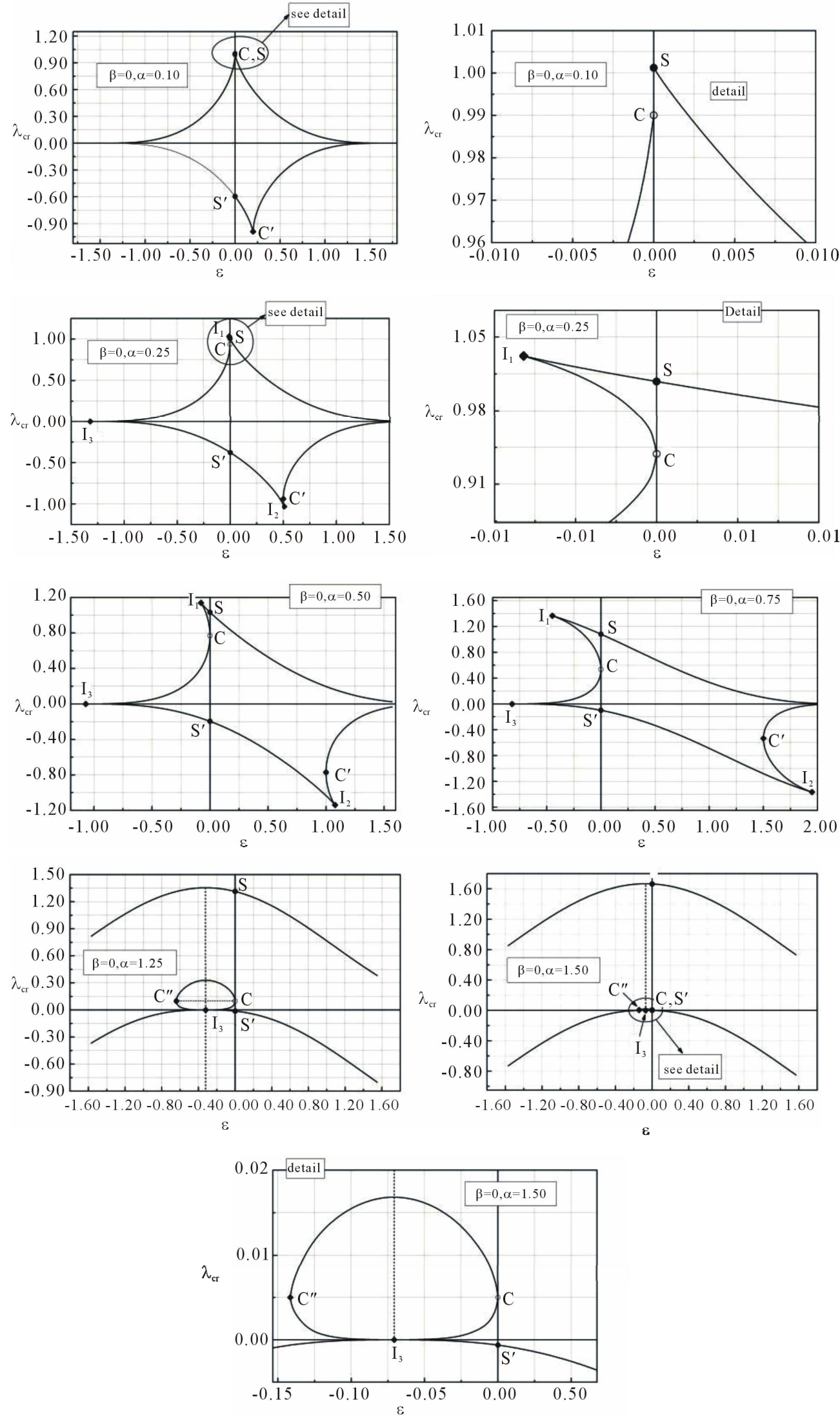

Figure 20. Diagrams of perturbed bifurcations and details for Case 5 and $\alpha<\pi / 2$. 

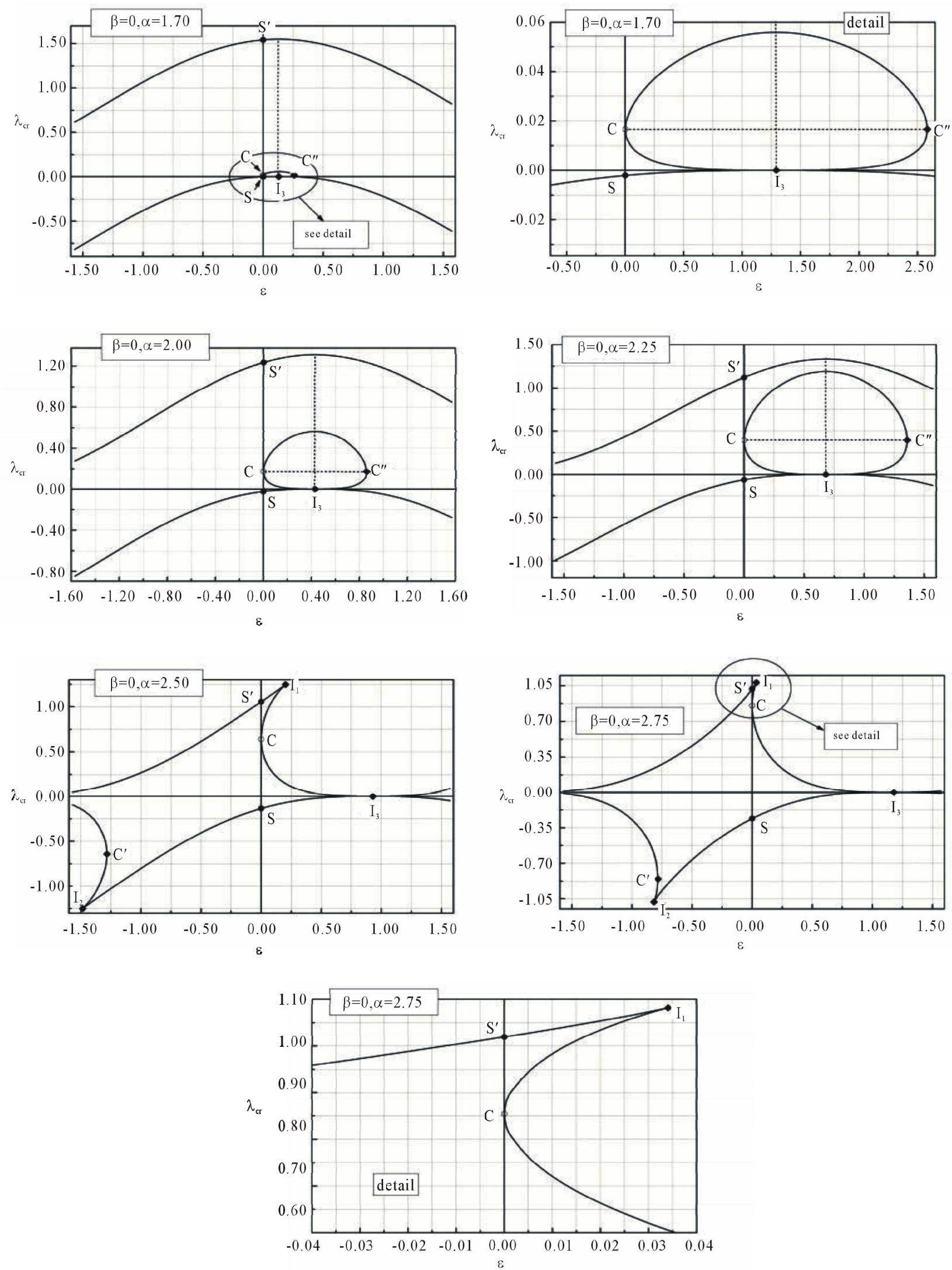

Figure 21. Diagrams of perturbed bifurcations and details for Case 5 and $\alpha>\pi / 2$. 

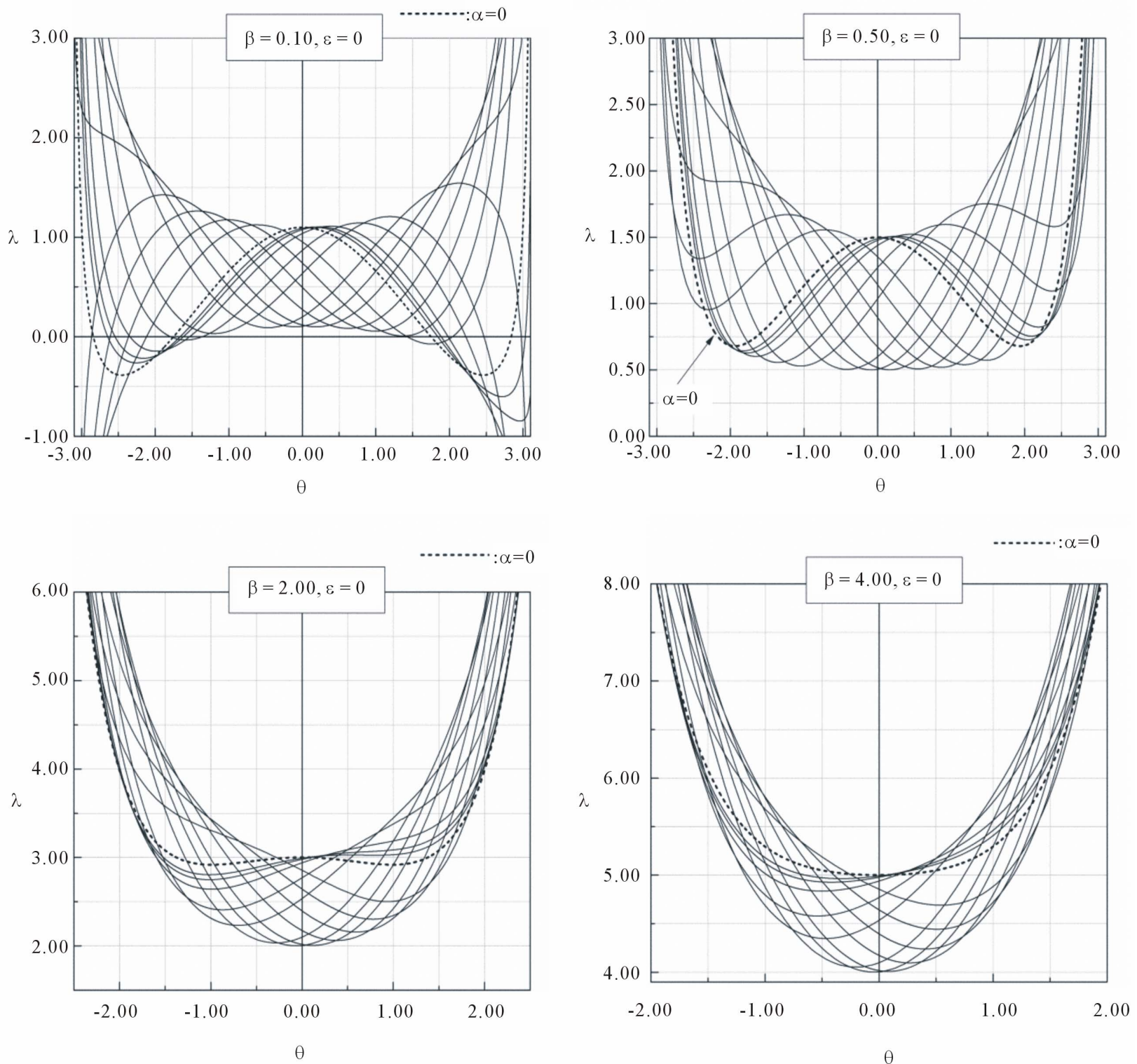

Figure 22. Equilibrium paths of Case 6, for specific values of $\beta$ and for $\alpha$ varying from 0 to $\pi / 2$.

$$
\lambda_{C}=\frac{1}{2}(1+2 \beta+\cos 2 a) .
$$

which is equal to $\beta+1$ for $a=0$ (as in relation (13)) and equal to

$$
\frac{1}{2}(1+\cos 2 \alpha)=\cos ^{2} \alpha \text { for } \beta=0
$$

(as in relation (18)). Except this bifurcation point, the equilibrium paths exhibit also other critical points as well.
These are limit points that occur at values of $\beta$ evaluated by zeroing the 2 nd variation of the potential. These values are given by

$$
\beta_{S}=\frac{\csc \theta(2 \sin 2 a+\sin [2 a-3 \theta]-3 \sin [2 a-\theta])}{4-4 \theta \cot \theta} .
$$

which for $\alpha=0$ yield the corresponding ones of Case 1 from relation (14). Hence, the locus of the limit points is expressed by

$$
\lambda_{S}=\frac{-2+4 \csc \theta\left(\theta \cos [2(a-\theta)]+\theta \sin a \sin [a-\theta]-\cos \left[2 a-\frac{3 \theta}{2}\right] \sin \frac{\theta}{2}\right)}{-4+4 \theta \cot \theta}
$$


Its graph is also depicted in Figure 23.

More specifically, as far as the limit points and their
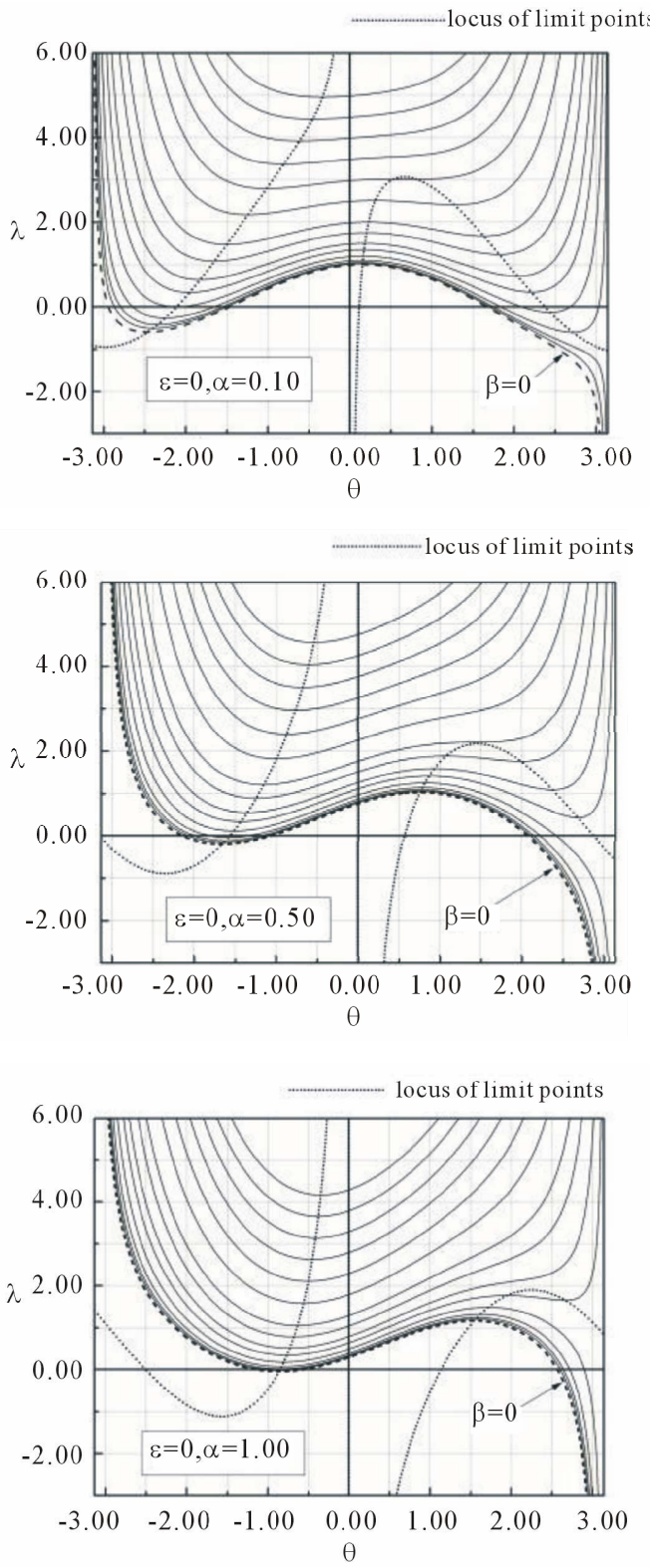

emergence or degeneration are concerned, the following can be observed:
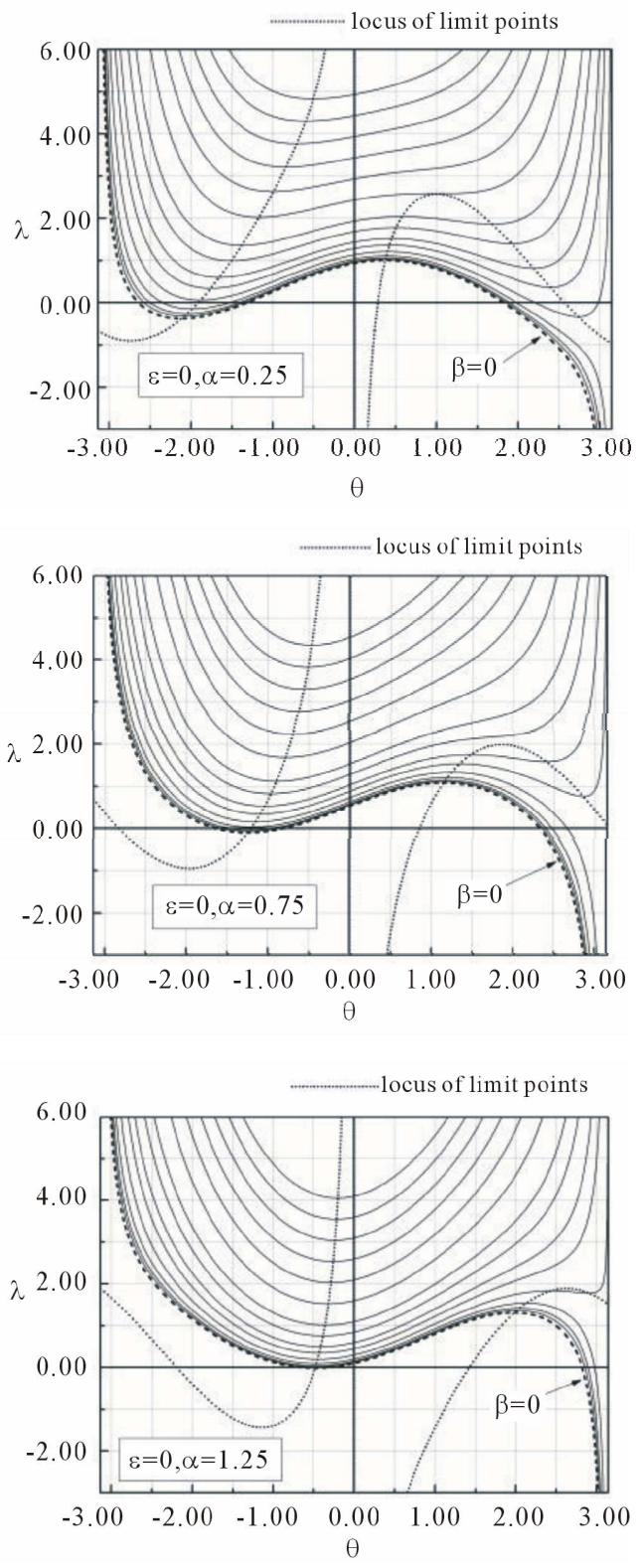

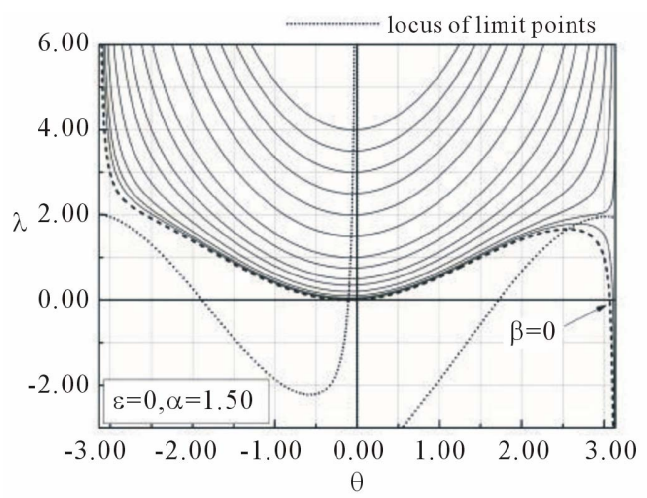

Figure 23. Equilibrium paths of Case 6, for specific values of $\alpha$ and varying $\beta$. 
1) For $\alpha<\pi / 2$ and for every $\beta$, the system always exhibits a limit point for negative values of $\theta$, and as $\beta$ increases the limit point load tends asymptotically to $+\infty$ and the corresponding angular deformation $\theta$ tends to zero. For limit points associated to positive $\theta$, it is valid that for relatively small $\beta$ only one limit point exists, being perturbation of the corresponding limit point of the system with $\beta=0$. As $\beta$ increases further, the system experiences a 2nd limit point, that can be numerically evaluated, which eventually coincides with the 1 st limit point at a critical values of $\beta=\beta_{c r}$, associated with a cutoff point of the equilibrium path. For larger values of $\beta$ these limit points seize to exist.

2) For $\alpha>\pi / 2$ similar phenomena occur, but for op-
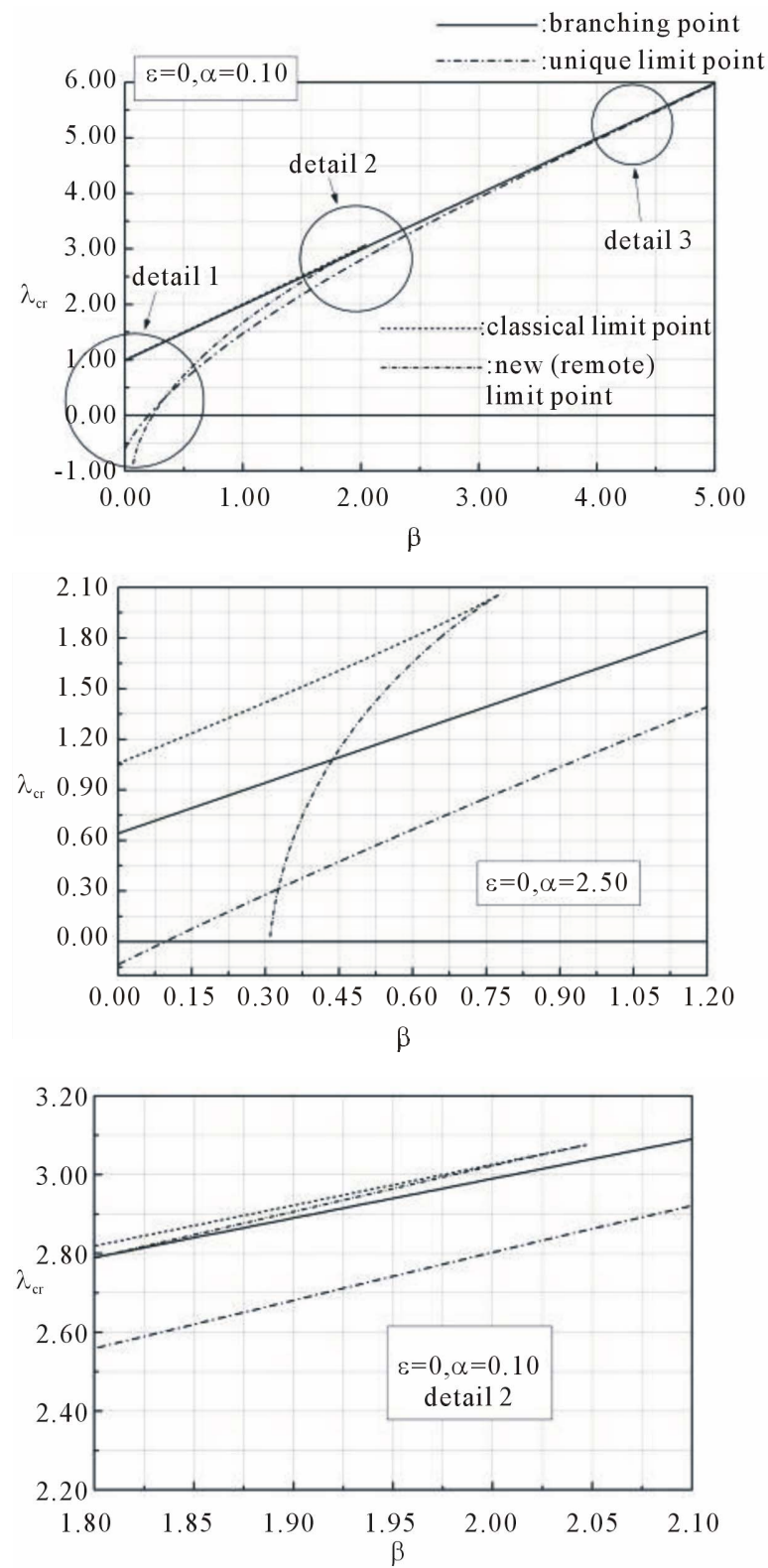

posite semi-planes with respect to the loading-axis, i.e. for $\theta$ of opposite sign.

Regardless however of $\alpha$ being small or greater than $\pi / 2, \beta_{c r}$ may be evaluated by solving numerically the system of equations

$$
\left\{\lambda_{E}=\lambda_{S}, \frac{\mathrm{d} \lambda_{S}}{\mathrm{~d} \theta}=0\right\} .
$$

with respect to $\theta$ and $\beta$, using the expressions given in Equations (23b) and (26). One may then draw bifurcation diagrams of the system for specific values of $\alpha$ and varying $\beta$, such as the exemplary ones illustrated in Figure 24.
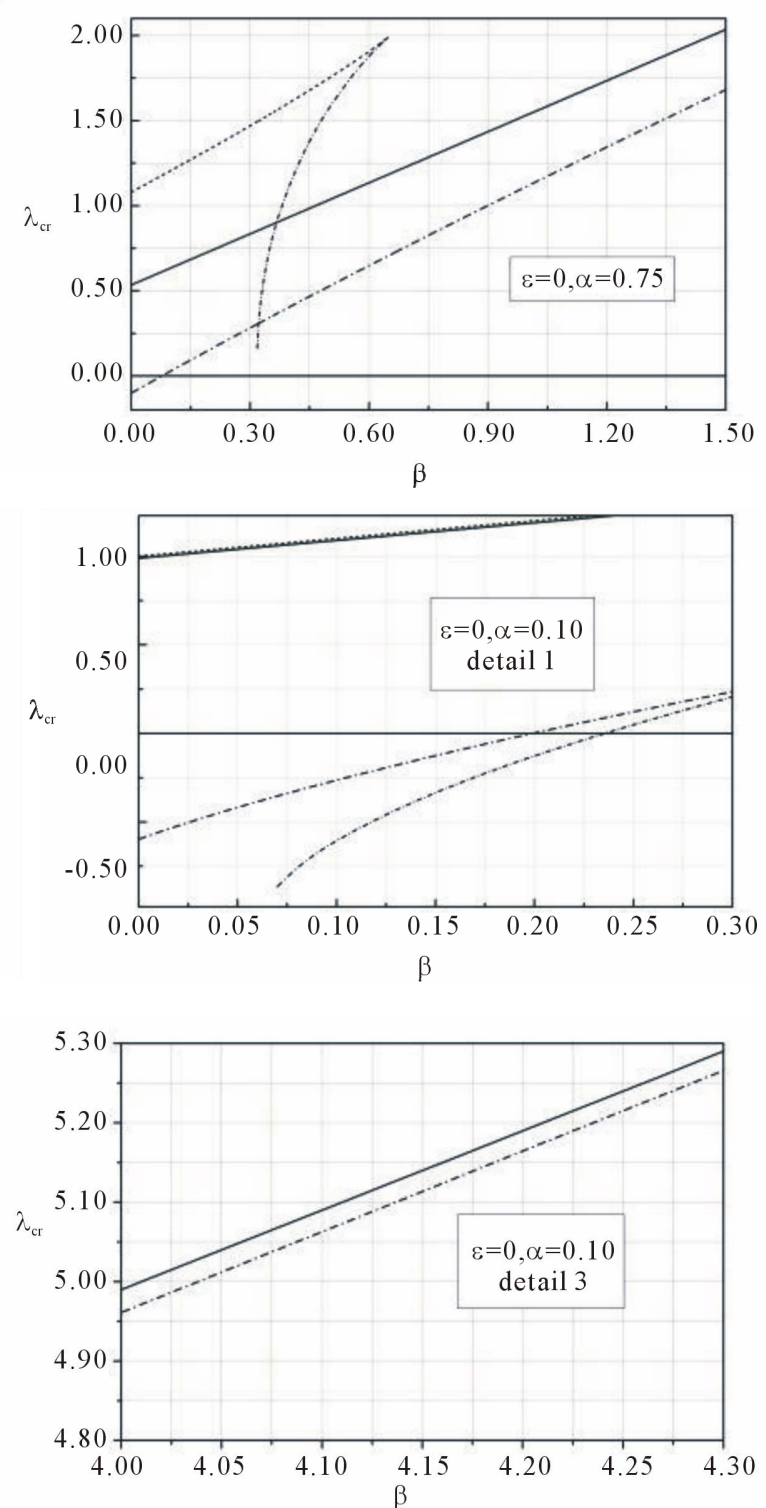

Figure 24. Bifurcation diagrams and details of system Case 6, for four values of $\alpha$. 
Although no imperfection is introduced, there exist tilted cusps, birth and vanishing of critical points as well as shifts and rotations of paths. These facts indicate a much more complicated structure of the equilibria than expected, which is attributed to the inclusion of $\alpha$ in the control parameters.

\subsection{Case 7: $\varepsilon, \alpha, \beta \neq 0$}

Up to now, the Cases considered could be studied rather comprehensively via standard procedures of nonlinear stability and bifurcation theory, since they involve maximum three control parameters and the visualization of results was to a significant extent satisfactory and quite informative, both quantitatively and qualitatively. However, in this Case with four control parameters (as well as to some extent for the Cases already studied with three control parameters) only ruled surface projections of equilibria and critical states on specific parameter planes may lead to proper visualizations [10], seen from a Ca- tastrophe Theory point of view. This leaves us only with limited opportunities when using Nonlinear Stability Theory but nevertheless some indicative results may be produced. Within the scope of this work, we will demonstrate the diagram of perturbed bifurcation of a system with $\alpha=0.50, \beta=0.75$ and varying the initial imperfection $\varepsilon$. The equilibrium paths and the critical points of the corresponding perfect system are illustrated in Figure 25, the data for its critical points are given in Table $\mathbf{1}$ and the aforementioned diagram in Figure 26.

The above diagram illustrates a typical situation arising in the butterfly singularity, which indicates that the system is associated with this catastrophe type. Analysis and proof of this fact are given in a companion paper.

\section{Concluding Remarks}

From the nonlinear stability analysis of the single-degree-of freedom mechanical model dealt with, it is concluded that all distinct critical points can be exhibited,

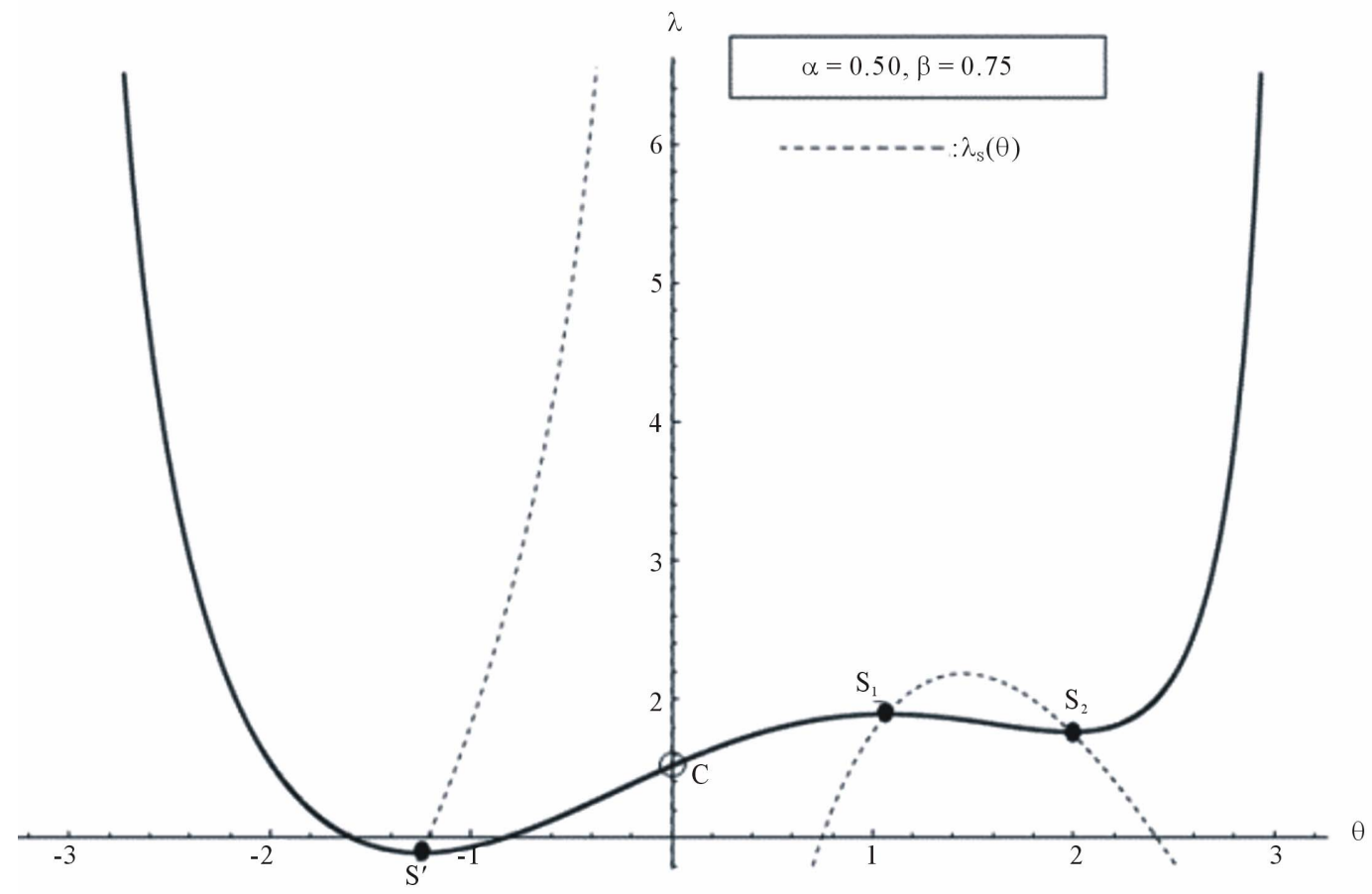

Figure 25. Equilibrium path and critical points for the perfect system with $\alpha=0.50, \beta=0.75$ (belonging to Case 6 ).

Table 1. Data of critical points of the perfect system with $a=0.50, \beta=0.75$.

\begin{tabular}{cccc}
\hline Critical point & Characterization & Angle $\theta$ & $\operatorname{Load} \lambda$ \\
\hline$C$ & Asymmetric branching point & 0 & 1.52015 \\
$S_{1}$ & Limit point & 1.0701 & 1.89289 \\
$S_{2}$ & Limit point & 1.98732 & 1.76441 \\
$S^{\prime}$ & Limit point & -1.24749 & 0.893122 \\
\hline
\end{tabular}




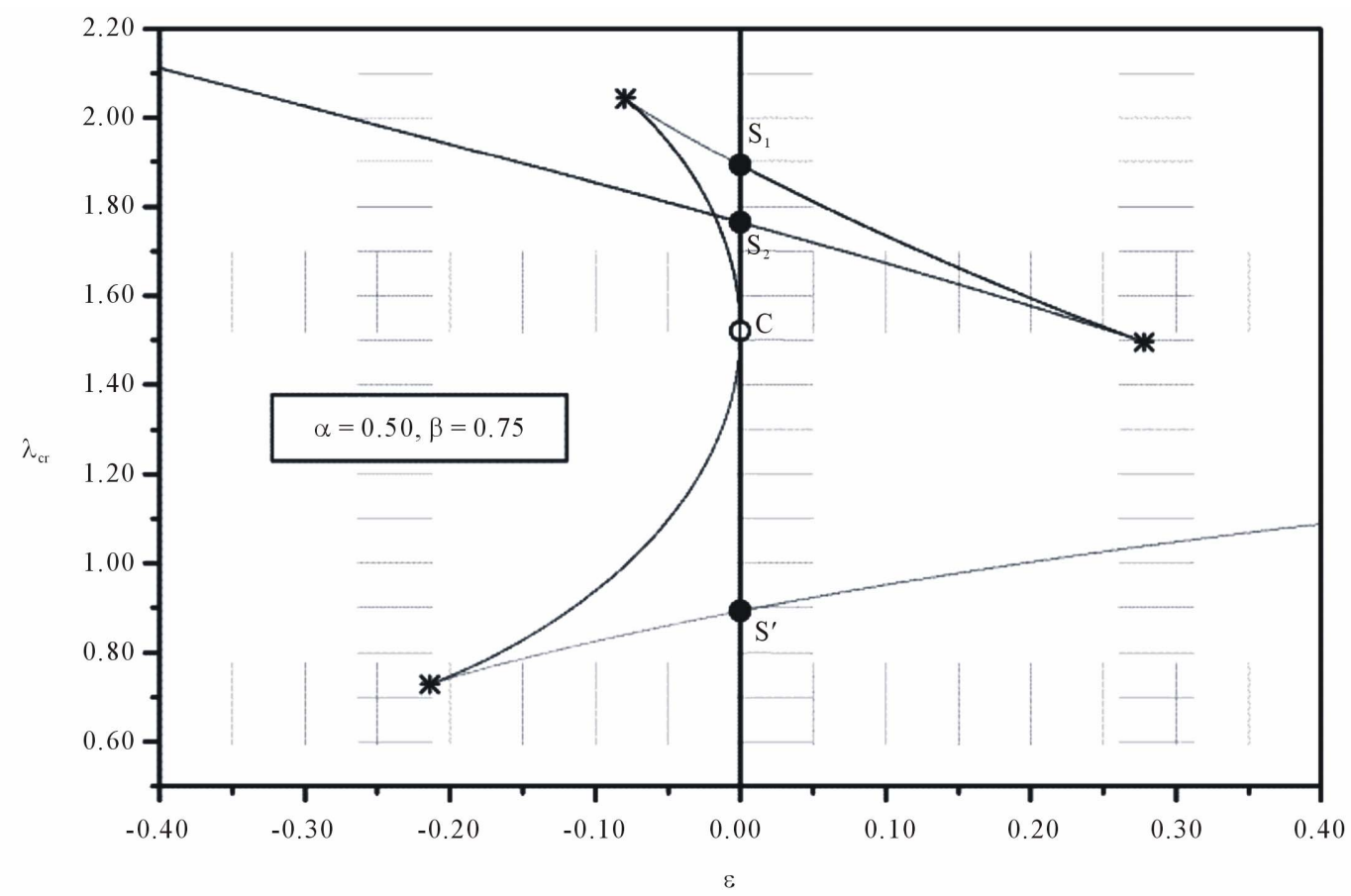

Figure 26. Bifurcation diagram for the system with $a=0.50, \beta=0.75$ and varying $a$.

whose evolution, as more control parameters are introduced, is associated with vanishing, birth of new critical points, hysteresis points, cusps and tilted cusps and other singularities. Behavior closely related to the swallowtail and butterfly catastrophes are reported and the system, although rather simple in nature, requires also the application of catastrophe theory for a full understanding of its stability response. This will be given in a companion paper.

\section{REFERENCES}

[1] G. W. Hunt, "Discrete Branching Points in the General Theory of Elastic Stability," Journal of the Mechanics and Physics of Solids, Vol. 13, No. 5, 1965, pp. 295-310. doi:10.1016/0022-5096(65)90033-5

[2] J. M. T. Thompson and G. W. Hunt, "Elastic Instability Phenomena," John Wiley \& Sons Ltd., Chichester, 1984.

[3] L. Godoy, "Theory of Elastic Stability: Analysis and Sensitivity," Taylor \& Francis, Philadelphia, 1999.

[4] G. W. Hunt, "The Discrete Branching Point as a One Degree of Freedom Phenomenon," International Journal of Solids and Structures, Vol. 7, No. 5, 1971, pp. 495-503. doi:10.1016/0020-7683(71)90101-6

[5] H. Troger and A. Steindl, "Nonlinear Stability and Bifurcation Theory," Springer, New York, 1991. doi:10.1007/978-3-7091-9168-2

[6] R. Gilmore, "Catastrophe Theory for Scientists and Engineers,” Dover Publ. Co., New York, 1981.

[7] D. S. Sophianopoulos, "Bifurcations and Catastrophes of a Two-Degrees-of-Freedom Nonlinear Model Simulating the Buckling and Postbuckling of Rectangular Plates," Journal of The Franklin Institute, Vol. 344, No. 5, 2007, pp. 463-488. doi:10.1016/j.jfranklin.2006.02.012

[8] V. Gioncu and M. Ivan, "Theory of Critical and Postcritical Behavior of Elastic Structures," Editure Academieie Republicii Socialiste, Romania, 1984.

[9] D. S. Sophianopoulos, "Static and Dynamic Stability of a Single-Degree-of-Freedom Autonomous System with Distinct Critical Points," Structural Engineering \& Mechanics, Vol. 4, No. 5, 1996, pp.529-540.

[10] A. E. R. Woodcock and T. Poston, "A Geometrical Study of the Elementary Catastrophes," Springer, New York, 1974. doi:10.1016/i.jfranklin.2006.02.012 